\title{
Dust Attenuation Curves in the Local Universe: Demographics and New Laws for Star- forming Galaxies and High-redshift Analogs
}

\author{
Samir Salim ${ }^{1}$ (1), Médéric Boquien ${ }^{2}$ (i), and Janice C. Lee ${ }^{3}$ (i) \\ ${ }^{1}$ Department of Astronomy, Indiana University, Bloomington, IN 47404, USA; salims@indiana.edu \\ ${ }^{2}$ Unidad de Astronomía, Facultad de Ciencias Básicas, Universidad de Antofagasta, Avenidad Angamos 601, Antofagasta, Chile \\ ${ }^{3}$ Spitzer Science Center, Caltech, Pasadena, CA 91125, USA \\ Received 2018 February 8; revised 2018 April 13; accepted 2018 April 16; published 2018 May 17
}

\begin{abstract}
We study the dust attenuation curves of 230,000 individual galaxies in the local universe, ranging from quiescent to intensely star-forming systems, using GALEX, SDSS, and WISE photometry calibrated on the Herschel ATLAS. We use a new method of constraining SED fits with infrared luminosity (SED+LIR fitting), and parameterized attenuation curves determined with the CIGALE SED-fitting code. Attenuation curve slopes and UV bump strengths are reasonably well constrained independently from one another. We find that $A_{\lambda} / A_{V}$ attenuation curves exhibit a very wide range of slopes that are on average as steep as the curve slope of the Small Magellanic Cloud (SMC). The slope is a strong function of optical opacity. Opaque galaxies have shallower curves-in agreement with recent radiative transfer models. The dependence of slopes on the opacity produces an apparent dependence on stellar mass: more massive galaxies have shallower slopes. Attenuation curves exhibit a wide range of UV bump amplitudes, from none to Milky Way (MW)-like, with an average strength one-third that of the MW bump. Notably, local analogs of high-redshift galaxies have an average curve that is somewhat steeper than the SMC curve, with a modest UV bump that can be, to first order, ignored, as its effect on the near-UV magnitude is 0.1 mag. Neither the slopes nor the strengths of the UV bump depend on gas-phase metallicity. Functional forms for attenuation laws are presented for normal star-forming galaxies, high- $z$ analogs, and quiescent galaxies. We release the catalog of associated star formation rates and stellar masses (GALEX-SDSS-WISE Legacy Catalog 2).
\end{abstract}

Key words: dust, extinction - galaxies: fundamental parameters

\section{Introduction}

A dust attenuation curve (or a law) describes how a galaxy's integrated luminosity arising from stellar and nebular continua is affected by internal dust at different wavelengths: from far-ultraviolet (UV), where the attenuation is usually the most severe, to near-infrared (IR), where it becomes mostly negligible. The knowledge and appropriate use of attenuation curves are critical for the study of galaxy populations and, consequently, of galaxy evolution. In particular, the knowledge of the attenuation curve is required for the robust derivation of a galaxy's physical parameters, especially in the absence of dust emission information from the mid- and/or far-IR. This is often the case for high-redshift galaxies (e.g., Bouwens et al. 2014; Oesch et al. 2014; Smit et al. 2014; Bowler et al. 2015; Finkelstein et al. 2015) and will be common for early-universe galaxy populations to be studied by the James Webb Space Telescope (JWST) (unless accompanied by observations made by ALMA). Attenuation curves serve as an input in galaxy simulations (e.g., Davé et al. 2017), constrain the physical properties of dust grains in different environments, inform radiative transfer models, and characterize dust-star distribution and geometry as a function of galaxy properties.

Though a great deal of progress has been made in the study of galaxy attenuation curves, there are many open questions (Conroy 2013). How diverse are attenuation curves from one galaxy to another? Are there trends between curve properties and the galaxy's physical and geometrical properties, such as the mass, star formation rate (SFR), or inclination?

That galaxies may exhibit a diversity of attenuation curves has its roots in the investigation of extinction curves, which precedes the work on attenuation curves by several decades. Extinction curves along individual sightlines are most commonly determined using the "pair method" (Stebbins \& Whitford 1943). Spectra or photometry of reddened stars are compared to the spectra or photometry of unreddened stars of the same spectral type. Extinction curves have been studied along multiple lines of sight to individual stars in the Milky Way (MW) and Large and Small Magellanic Clouds (LMC and SMC; Nandy et al. 1975, 1980; Rocca-Volmerange et al. 1981), from which the average total extinction curves currently in use for these galaxies have been derived (e.g., Seaton 1979; Prevot et al. 1984; Fitzpatrick 1986; Cardelli et al. 1989; Gordon et al. 2003). For galaxies other than the MW and the Clouds, the extinction curves have been studied only in special circumstances, such as in occulting pairs (e.g., White \& Keel 1992; Holwerda et al. 2013; Keel et al. 2014). Great diversity is seen both between different lines of sight in a single galaxy and between the average curves of the MW, LMC, and SMC. The diversity of curves can be characterized by two main features: their slope in the UV/optical wavelength range, and the presence or absence of additional absorption at $1700-2700 \AA$ (the near-UV (NUV) range), known as the $2175 \AA$ bump or just the UV bump (Stecher 1965). Extinction curves toward MW sightlines show a range of slopes with various degrees of UV bump strengths (Fitzpatrick \& Massa 1986, 1988; Cardelli et al. 1989). Steeper slopes tend to be associated with sightlines that do not penetrate deeply into the molecular clouds. Averaging the curves for lines of sight that pass only through diffuse dust, an average MW extinction curve is derived that nevertheless has a relatively shallow (gray) slope and quite pronounced UV bump. In contrast to the MW curve, the SMC extinction curve is significantly steeper (power-law exponent 
steeper by about 0.5 ), and most sightlines lack the UV bump (Bromage \& Nandy 1983). The LMC curve lies in between the MW and SMC curves in terms of steepness, as well as by having an intermediate UV bump. The drivers of these differences are currently not well known.

If attenuation curves were similar to extinction curves, one may think, based on just three galaxies, that massive galaxies have shallower and bumpier curves than lower-mass galaxies. However, an average extinction curve does not necessarily describe how the integrated light of a galaxy is affected by the dust, i.e., their attenuation curve. The latter includes, in addition to absorption and scattering out of the line of sight, the scattering into the line of sight and the effects of dust/star distribution and viewing orientation (the "geometry"; see the review by Calzetti 2001, also Charlot \& Fall 2000; Conroy et al. 2010; Chevallard et al. 2013; Conroy 2013). In addition to the possible variation as a function of galaxy mass, there is a question of whether starbursting galaxies, and by extension, high-redshift populations, differ from more normal starforming galaxies at low redshift.

There are two main methods for deriving attenuation curves: the "comparison method" and the "model-based method." They fundamentally differ in the way in which they establish the level of attenuation of galaxies. The comparison method does it empirically (usually based on the nebular emission Balmer decrement, which serves as a proxy for continuum attenuation), while the model-based method uses attenuated stellar population synthesis models. Furthermore, the comparison method produces an average curve for an aggregate of galaxies, whereas the model-based method produces curves for individual galaxies. Model-based methods have started to be exploited relatively recently.

The comparison method was first employed in local UVselected starburst galaxies (Calzetti et al. 1994, 2000). The motivation behind focusing on starbursts, a population of galaxies that is relatively rare in the local universe, was to provide guidance for the interpretation of the rest-frame UV emission of high-redshift $(z>1)$ populations (Lyman-break galaxies), which the local starburst resembles. Furthermore, the IUE UV spectra (Kinney et al. 1993) of starbursts had better constraining power because of the higher signal in UV spectra compared to the "normal" star-forming galaxies. The slope of the Calzetti ("starburst") attenuation curve is similar to that of the MW extinction curve (when normalized by $A_{V}$; see Section 3.4), i.e., it is shallow, but without the UV bump and with a somewhat less abrupt far-UV (FUV) rise. This result forms the basis for a picture in which local starburst galaxies and high-redshift populations have shallow, bump-free (i.e., Calzetti) curves, whereas more normal galaxies may or may not have either steeper or bumpier curves.

The attenuation curves of more normal SF galaxies have only recently been derived, but with conflicting results. The pairmatching method of Wild et al. (2011), which, like the comparison method, uses the Balmer decrement but does not explicitly require identification of unattenuated galaxies, was employed to derive the attenuation curve of a larger and more general population of local star-forming galaxies, showing a diversity of attenuation curves as a function of physical properties of galaxies, but yielding typical curves that were closer to the Calzetti curve than the steep SMC curve. Battisti et al. (2016, 2017) extended the Calzetti comparison method to normal star-forming galaxies (using UV/optical photometry instead of spectra), and found almost identical (shallow, no-bump) curves. On the other hand, studies that use model-based methods tend to find, on average, steeper slopes both locally (Conroy et al. 2010; Leja et al. 2017) and at higher redshift (Arnouts et al. 2013; Kriek \& Conroy 2013; Salmon et al. 2016; Reddy et al. 2018). Furthermore, many studies present evidence for a moderate UV bump both in local normal star-forming galaxies (Conroy et al. $2010)$ and to some extent in higher-redshift $(z>1)$ populations (Noll et al. 2007; Kriek \& Conroy 2013).

In this paper, we study the demographics of attenuation curves in the local universe in a more extensive way and further probe the issues regarding the diversity of the curves, the presence of the bump, and the extent of differences between different populations. We also present a discussion on why different methodologies may produce systematically different results, especially in terms of curve steepness.

We constrain individual dust attenuation curves for a very large sample of galaxies, spanning those that are quiescent to intensely star-forming. To overcome some of the challenges involved with this task, we apply a novel version of the energybalance SED-fitting method, where the constraints from the IR dust emission are applied in a way that is computationally practical and yet samples the parameter space with high resolution. Our study builds on previous efforts to constrain attenuation curves using model-based methods carried out on local galaxies but on smaller scale (Burgarella et al. 2005; Leja et al. 2017), or for larger samples but at higher redshifts (Arnouts et al. 2013; Kriek \& Conroy 2013; Salmon et al. 2016; Tress et al. 2018).

The current study also updates the GALEX-SDSS-WISE Legacy Catalog (GSWLC), a catalog of physical parameters of 700,000 galaxies in the local universe from the Bayesian SED fitting (Salim et al. 2016). The robustness of GSWLC parameters was the result of the careful treatment of input photometry, a wide range of physically motivated star formation histories, the inclusion of emission-line corrections, and importantly, appropriate choices regarding the dust attenuation curve. In this work, we expand on those efforts by directly including the IR constraints that further refine the SFRs. The sample and input data are described in Section 2. Details regarding the methodology of IR-luminosity determination, the SED fitting, and the parameterization of attenuation curves are given in Section 3. The resulting attenuation curves, their dependence on physical properties of galaxies, comparison with previous attenuation and extinction curves, and functional fits are presented in Section 4. Section 5 discusses our results in the context of previous studies, and Section 6 presents the summary of the results.

Throughout this work, we assume a Chabrier initial mass function (IMF; Chabrier 2003) and WMAP7 flat cosmology $\left(H_{0}=70.4 \mathrm{~km} \mathrm{~s}^{-1} \mathrm{Mpc}^{-1}, \Omega_{m}=0.272\right)$.

\section{Samples and Data}

We utilize UV and optical photometry together with the estimates of total IR luminosity to derive the total dust attenuation curves of a large number of individual galaxies. IR luminosities were obtained from the mid-IR photometry (12 or $22 \mu \mathrm{m}$ ), using luminosity-dependent IR templates and further corrected using the calibrations derived from a subset of galaxies that have far-IR photometry. The resulting IR luminosities have an accuracy of $\sim 0.1 \mathrm{dex}$, which, together with the UV and optical photometry, allows the slopes of 
individual attenuation curves to be determined with a typical error of 0.25 in the power-law slope exponent, which is several times smaller than the range of slope exponents.

Dust attenuation curves in this paper are determined for GSWLC-M galaxies that have mid-IR and UV photometry. GSWLC sample construction is described in detail in Salim et al. (2016). In short, GSWLC contains all galaxies with SDSS DR10 redshifts below $z=0.3$, brighter than $r_{\text {petro }}=18.0$, and covered by FUV and NUV observations from GALEX (data release GR6/7). Because GALEX observations span a wide range of depths, separate samples were produced, and independent SED fitting was performed for the shallow, "allsky" (GSWLC-A), medium-deep (GSWLC-M), and deep (GSWLC-D) UV surveys, which encompass 88\%, 49\%, and $7 \%$ of SDSS target galaxies, respectively. If a galaxy was covered by more than one UV survey, it was included in each of respective catalogs. Mid-IR observations (12 and $22 \mu \mathrm{m})$ from WISE cover the entire sky.

GSWLC-M balances UV depth and sample size, and so we base our target sample on it. GSWLC-M includes 358,121 galaxies, regardless of an IR or UV detection. For the purposes of this paper, we require a detection in either 12 or $22 \mu \mathrm{m}$ and in one UV band. When only one UV band yields a detection, it is invariably the NUV, which goes deeper than the FUV. Requiring both NUV and FUV would reduce the sample size but is not necessary because the slope, being parameterized, is relatively well constrained by the overall behavior at longer wavelengths. We verify that there are no systematic differences between single- and two-band results. There are 228,335 galaxies in our target sample after IR and UV detection cuts, with the mean redshift of 0.10 .

We will specially focus on $\sim 1 / 2$ of the sample classified as star-forming, i.e., the galaxies that lie below the (active galactic nucleus) AGN demarcation line of Kauffmann et al. (2003) in the Baldwin et al. (1981, BPT) emission-line diagram. We find that galaxies can be securely classified with the BPT diagram by requiring the signal-to-noise ratio $(\mathrm{S} / \mathrm{N})$ of the $\mathrm{H} \alpha$ line to be greater than 10 and the $\mathrm{S} / \mathrm{N}$ ratio of the other three lines to be greater than 2 . The usual $\mathrm{S} / \mathrm{N}>3$ cut is overly restrictive because the $\mathrm{S} / \mathrm{N}$ ratio of line ratios of closely separated lines is actually higher than the $\mathrm{S} / \mathrm{N}$ ratio of individual lines (Juneau et al. 2014). More accurately, this selection removes galaxies with significant AGN contribution (even if some may have relatively high levels of SF) and galaxies with weak emission lines. The resulting "star-forming" sample contains 113,892 galaxies with sSFR $>-11$, i.e., it contains most of the galaxies that form the star-forming sequence (galaxy "main sequence").

In this paper, we also utilize the calibration sample, composed of galaxies from Herschel ATLAS Data Release 1. Herschel ATLAS is the most extensive deep far-IR survey, covering $161 \mathrm{deg}^{2}$ corresponding to three fields in the GAMA spectroscopic survey (Valiante et al. 2016), which is itself located within the SDSS footprint. Specifically, we use catalog version 1.2, from which we extract Herschel ATLAS galaxies included in the SDSS DR10 spectroscopic survey (based on the Bourne et al. 2016 matching), having $z<0.3, r_{\text {petro }}<18.0$ (same cuts as GSWLC), and detected at $22 \mu \mathrm{m}$ with WISE. Furthermore, we require that calibration galaxies be classified as star-forming (i.e., without significant AGNs based on the BPT diagram) in order to remove the potential contamination by nonstellar dust heating. The resulting Herschel-WISESDSS calibration sample contains 1891 galaxies and is a representative subsample of the target sample, having the same distribution in the $\mathrm{SSFR}-M_{*}$ plane and the same range of IR luminosities (Section 3.1).

For the target sample, we use UV photometry from GALEX, optical photometry from SDSS, and mid-IR photometry from WISE. We apply various corrections to the FUV and NUV photometry to correct for the edge-of-the-detector effects and blending, as described in Salim et al. (2016). Five-band SDSS photometry uses modelMag magnitudes. UV and optical fluxes are corrected for Galactic reddening using prescriptions from Peek \& Schiminovich (2013) and Yuan et al. (2013), respectively.

For the target and calibration samples, we use photometry from two longer-wavelength WISE channels: W3 and W4, centered at 12 and $22 \mu \mathrm{m}$, respectively. Photometry is taken from the unWISE catalog (Lang et al. 2016), which performed forced photometry on WISE images using SDSS centroids and profiles as priors. For a sample that is a mix of galaxies that are resolved and unresolved in WISE, the prior-based photometry is more appropriate and less biased than the PSF magnitudes from the official AllWISE Source Catalog.

Far-IR and submillimeter (sub-mm) photometry from Herschel used for the calibration sample consists of 100 and $160 \mu \mathrm{m}$ fluxes from the PACS instrument, and 250, 350, and $500 \mu \mathrm{m}$ fluxes from the SPIRE instrument, and is described in Valiante et al. (2016).

To derive gas metallicities, AGN strength, and BPT classification, we utilize emission-line fluxes and equivalent widths from the MPA/JHU catalog. These data, based on SDSS DR7 spectroscopic reductions (Tremonti et al. 2004), are available for $97 \%$ of target sample galaxies.

\section{Methodology \\ 3.1. Derivation of IR Luminosities}

In this paper, we use a novel approach to energy-balance SED fitting. In short, instead of fitting an SED that includes both stellar (UV/optical) and dust (IR) SEDs, we perform UV/ optical SED fitting that includes constraints on the dust emission directly from the total IR luminosity, i.e., the IR luminosity is treated as a "flux" point. This approach, which we call SED+LIR fitting, enables more robust results with a larger number of model SEDs. Details of the method are discussed in Section 3.2.

To obtain IR luminosities for our target sample, which typically only has mid-IR photometry (from WISE), we perform a two-step process. In Step 1, we derive an estimate for the IR luminosity based on a single flux point $(22 \mu \mathrm{m}$, if detected, or $12 \mu \mathrm{m}$ otherwise), using the luminosity-dependent IR templates of Chary \& Elbaz (2001). For galaxies that belong to the Herschel calibration sample, we compare these estimates with the "true" IR luminosity obtained from the full IR SED and derive corrections (calibration) to be applied to Step 1 estimates. True luminosities are referred to as such because they incorporate the far-IR/sub-mm fluxes from Herschel in addition to the $22 \mu \mathrm{m}$ flux from WISE and are determined by fitting Chary \& Elbaz (2001) templates (irrespective of the IR luminosity associated with each template) to up to six flux points (one WISE, two PACS, and three SPIRE) and using $\chi^{2}$ minimization to find the best-fitting template and its scaling factor. The scaling factor is applied to the luminosity of the template to arrive at the final IR luminosity. The true 


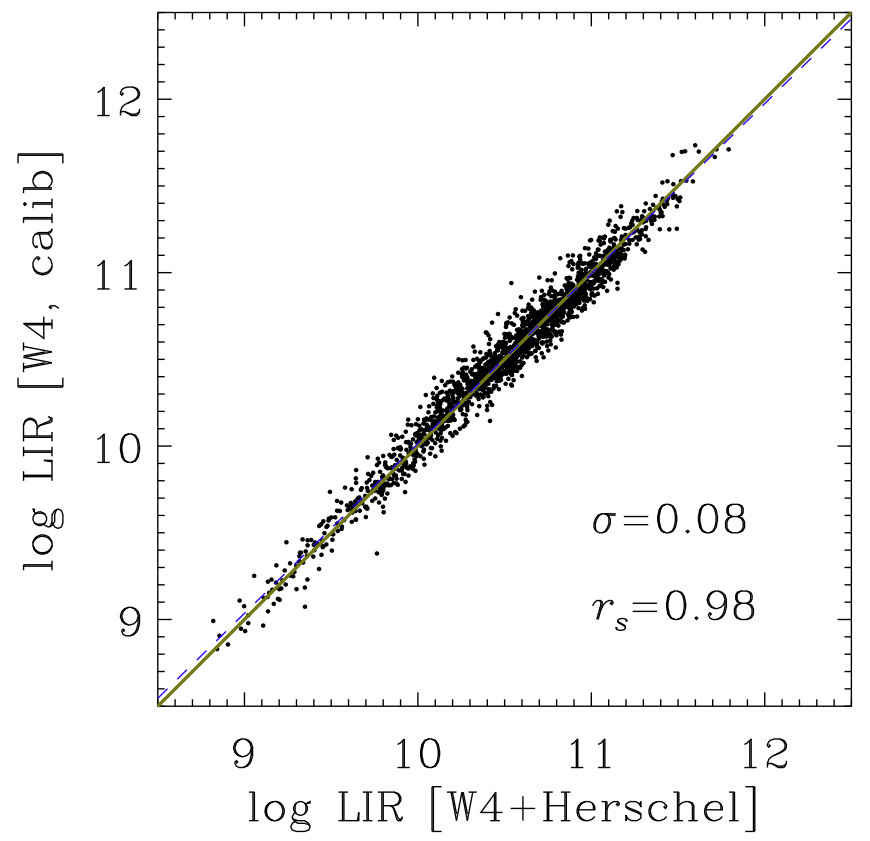

Figure 1. Comparison between the IR luminosity derived from just the mid-IR flux point $(22 \mu \mathrm{m}$, WISE channel W4), using the new calibration described in the text, and the IR luminosity derived from the full IR SED from Herschel (PACS and SPIRE data in combination with W4). Our procedure is based on the use of luminosity-dependent templates plus the application of corrections and results in estimates that have a small dispersion and no offset with respect to the full IR SED values. The green solid line is the 1:1 line, whereas the blue dashed line is the robust linear fit. Values of the standard deviation and the correlation coefficient are given in the plot.

luminosities of the calibration sample span three orders of magnitude, $8.8<\log L_{\mathrm{IR}}<11.8$. Furthermore, their distribution in the sSFR- $M_{*}$ diagram is essentially the same as that of the full sample to which the calibration is applied, with the average sSFRs being only 0.1 dex higher. In Step 2, we apply the corrections to IR luminosities of galaxies in the target sample. This method allows us to obtain accurate (step 2) IR luminosities without far-IR photometry. The accuracy is 0.08 (0.11) dex for luminosities based on 22 (12) $\mu \mathrm{m}$ flux, with the average systematic offset smaller than 0.01 dex. The accuracy is similar across all luminosities. The comparison between the IR luminosities derived from just the $22 \mu \mathrm{m}$ data and the full IR is shown in Figure 1. The accuracy of our two-step method is remarkable considering that the luminosities are ultimately based on a single flux point in the mid-IR. A detailed description of the technique and calibrations will be presented in a separate publication.

While most of the analysis in this paper focuses on galaxies selected as star-forming, we perform the SED fitting and derive dust attenuation parameters for non-star-forming galaxies as well, which include the galaxies classified as AGNs. The IR luminosities of AGN may be affected by nonstellar dust heating, especially since they are based on the mid-IR data. For galaxies classified as AGNs, we find a systematic trend between the SFRs from GSWLC-1 (as we will refer to the original version of the catalog), which were based solely on the stellar emission, and the SFRs from a simple conversion from the $22 \mu \mathrm{m}$-based IR luminosity (Salim et al. 2016). The trend increases with the equivalent width of the [O III]5007 line, a proxy for AGN strength (Kauffmann et al. 2003). Based on this trend, we derive a correction that is applied to the IR luminosities of the target sample galaxies classified as AGNs and is used in the SED fitting.

\subsection{Energy-balance SED Fitting}

This paper uses the Code Investigating GALaxy Emission (CIGALE; Noll et al. 2009, M. Boquien et al. 2018, in preparation) to perform the SED fitting and determine the physical properties of galaxies, such as the stellar mass and the current SFR, but also, importantly, the dust attenuation curve. In the construction of GSWLC-1 (Salim et al. 2016), we have performed the SED fitting of only the UV and optical photometry, i.e., the stellar and nebular emission. No constraints from the dust emission in the IR were used. Instead, we have relied on the bulk comparison between IRspecific SFRs (sSFRs; derived using a simple conversion of IR luminosities to SFR) and the SED-fitting-derived sSFRs to help us select dust attenuation curves to use in the UV/optical SED fitting. In particular, we have found that a good agreement between the UV/optical and IR sSFRs is achieved only if we assume an attenuation curve that is significantly steeper than the one given in Calzetti et al. (2000), with further improvements in the level of agreement when allowing the UV bump to be present in the curve (Figure 4 in Salim et al. 2016).

In this work, the IR luminosity is used in the SED-fitting explicitly, by applying a novel variant of energy-balance SED fitting. IR luminosity allows us to let the parameters that describe the attenuation curve be free and determine then from the fitting. Energy-balance SED fitting is the SED fitting that, in addition to stellar emission, also takes into account dust emission in the IR, such that the energy emitted by the dust (the IR luminosity) matches the energy absorbed by the dust in the UV through near-IR. In previous works, energy-balance SED fitting involved modeling both the stellar (UV to near-IR) and dust (mid- and far-IR) SEDs. Notably, such an approach is used in the SED-fitting code MAGPHYS (da Cunha et al. 2008), where the IR SED is modeled as a composite of PAH template spectra, the mid-IR continuum, and warm and cold grain emission components. This model of the IR SED requires six parameters, each with a range of values, resulting in a large number of IR SEDs alone (50,000 in the case of MAGPHYS), each of which must be considered in combination with a similar number of models describing the stellar emission, leading to 2.5 billion model SEDs. Even if additional constraints are used (e.g., that the sSFR and the IR SED shape are correlated; da Cunha et al. 2008) the approach of simultaneously modeling stellar and dust emission results in a very large number of parameter combinations. A similar approach was implemented in CIGALE, where the specification of dust emission leads to a proliferation of SED models that must be considered in the SED fitting. To keep the energybalance SED fitting computationally manageable while allowing the parameters that describe the dust attenuation curve to be unconstrained (which requires the number of models to be increased by a factor of $\sim 50$ - the number of different slope/ bump combinations), it would have been necessary to resort to a coarse sampling of the parameter space, potentially compromising the precision of the derived parameters.

This paper takes another approach that allows us to keep the same high-resolution model grid as the one used for just the UV/optical SED fitting. We do so by decoupling the determination of the IR luminosity from the SED fitting. 
Namely, we have neither the data nor need to fit for the shape of the IR SED, since what constrains the SFR and the attenuation curve is just the total IR luminosity. As shown in Section 3.1, by using luminosity-dependent IR templates together with the empirical corrections, we can obtain accurate IR luminosities from a single flux point. The key to our approach is to use the IR luminosity as a direct constraint in the SED fitting, without fitting the IR SED. We implement this approach using a custom modification of CIGALE v0.11, whereby the observed IR luminosity is treated as another SED "flux" point, i.e., the agreement between the dust luminosity predicted by the model and the observed IR luminosity goes into the $\chi^{2}$ that characterizes the quality of the fit. In this way, we obtain all of the benefits of the energy-balance SED fitting without the need to proliferate the number of models or be potentially biased from the uncertainties stemming from the limited coverage in the IR. We refer to this novel variant of energy-balance SED fitting as IR-luminosity-constrained SED fitting (SED+LIR fitting).

Next, we provide an overview of other aspects of SED fitting, with full details given in Salim et al. (2016). We use the same two-component exponential model for star formation histories as used in GSWLC-1, a parameterization consisting of an old population (formed $10 \mathrm{Gyr}$ before the present epoch) declining exponentially with varying $e$-folding times, and a younger population (100 Myr to $5 \mathrm{Gyr}$ old) having a mass fraction between zero and $50 \%$ of the old population and a nearly constant SFR. Such parameterization, yielding 1428 distinct SF histories, overcomes the limitations of singleexponential decline models (" $\tau$ models"), which, in order to produce high sSFRs (blue colors), must be made artificially young and hence may miss the mass from an old, fainter population (the "outshining" bias). The models are calculated for four stellar metallicities $\left(0.2-2.5 Z_{\odot}\right)$ using Bruzual \& Charlot (2003) single stellar populations. The use of Maraston (2005) models makes the resulting dust attenuation curves steeper by 0.1 . We use a Chabrier IMF but confirm that the derived values of dust attenuation parameters do not change beyond a few percent on average if the Salpeter IMF is used instead.

An important feature of CIGALE is that it allows the calculation of the contribution of emission lines to model broadband fluxes, the omission of which leads to an additional 0.1 dex noise in SFR determinations and a 0.3 dex overestimate in the determination of SFRs of high-sSFR galaxies (Figure 5 in Salim et al. 2016). Compared to GSWLC-1, we now use new, improved emission-line models with a more extended range and finer resolution of ionization parameters. The emission lines are computed from the number of ionizing photons emitted by stellar populations. The nebular templates expand upon Inoue (2011) and have been computed using Cloudy 08.00 (Ferland et al. 1998). They include the nebular continuum (free-free, free-bound, and two-photon processes) as well as 124 emission lines. The electron density is set to $100 \mathrm{~cm}^{-3}$. Furthermore, we now select both the ionization parameter $(\log U=-3.4)$ and the fraction of Lyman continuum photons absorbed by dust $\left(f_{\text {dust }}=0.3\right)$, such that the model equivalent widths of the main optical lines $(\mathrm{H} \alpha, \mathrm{H} \beta$, [O II], [O III]) match, on average, the observed equivalent widths from SDSS spectra. Our adopted fraction agrees with the estimates in the literature (Inoue 2001; Iglesias-Páramo et al. 2004), although we find that assuming a larger or smaller value has essentially no effect on the results.

\subsection{Dust Attenuation Curve Nomenclature}

Dust attenuation curves have been formulated in three different ways in the literature, depending on the adopted normalization and whether or not the curve is relative. It is useful to review these definitions and the corresponding nomenclature. We refer to the three formulations as the selective, total, and absolute attenuation curves, which in their most fundamental form can be expressed as

$$
\begin{array}{cc}
\text { Selective: } & \frac{A_{\lambda}-A_{V}}{A_{B}-A_{V}}, \\
\text { Total: } & \frac{A_{\lambda}}{A_{B}-A_{V}}, \\
\text { Absolute: } & \frac{A_{\lambda}}{A_{V}},
\end{array}
$$

where the attenuation of the continuum at some wavelength, $A_{\lambda}$, is directly related to the optical depth. Two galaxies can have the same attenuation law, with one having a higher $A_{\lambda}$ than the other, meaning it is dustier. Thus, in order to obtain the attenuation curve, $A_{\lambda}$ must be normalized. In selective and total formulations, the normalization is done by the color excess $E(B-V) \equiv A_{B}-A_{V}$. The total formulation of the attenuation curve is often denoted as $k_{\lambda}$ :

$$
k_{\lambda}=\frac{A_{\lambda}}{E(B-V)}=\frac{A_{\lambda}}{A_{B}-A_{V}} .
$$

The value of the total attenuation curve in the $V$ band is $k_{V}=A_{V} / E(B-V) \equiv R_{V}$, and is known as the ratio of total to selective extinction in $V$. For the MW and LMC extinction curves, it is customary to use $R_{V}=3.1$, an average of different sightlines through the diffuse ISM, having a range of values between 2 and 6 (Cardelli et al. 1989). The Calzetti curve is associated with $R_{V, \mathrm{Cal}}=4.05$, with a galaxy-to-galaxy scatter of 0.8 (Calzetti et al. 2000). The selective formulation has mostly been used in the older literature, where the total curve could not be obtained because of the lack of IR data needed to anchor the curve. The selective curve (sometimes designated as $E(\lambda-V) / E(B-V))$ is related to the total curve as $k_{\lambda}-R_{V}$, i.e., it only gives the relative attenuation with respect to $V .{ }^{4}$

The third way to formulate the attenuation curve is to normalize $A_{\lambda}$ by the attenuation in $A_{V}$. This form is related to the total curve formulation through

$$
A_{\lambda} / A_{V}=k_{\lambda} / k_{V}=k_{\lambda} / R_{V} .
$$

Normalization by absolute attenuation $\left(A_{V}\right)$ is more intuitive (and more fundamental; Cardelli et al. 1989) than by color excess because the steepness of the attenuation curve in different parts of the spectrum has a direct interpretation. (By contrast, in the total formulation, the slope of the curve between $B$ and $V$ is identical by definition). Furthermore, it is this form that is required and sufficient to model the dust attenuation of galaxy spectra. For historical reasons, the normalization of the

\footnotetext{
4 The selective attenuation curve is what Reddy et al. (2015) refer to as $f Q(\lambda)$, with $Q(\lambda)$ being the non-normalized selective curve introduced in Calzetti et al. (1994).
} 
curve is tied to the $V$ band, though ideally it should be at longer wavelengths, because different sightlines in our galaxy still show some variations in the extinction curves around $V$ (Cardelli et al. 1988). This formulation, which, following Cardelli et al. (1988) we refer to as the absolute, will be used to discuss the results in this paper. Thus, whenever we refer to a curve being steeper than another, we will mean steeper in $A_{\lambda} / A_{V}$

Note that two curves that have the same slopes in total formulation but are offset from one another (i.e., have different $R_{V}$ ) will not have the same slopes in absolute formulation (and thus will not affect the light in the same way). Lower values of $R_{V}$ mean that for the same $A_{V}$ the attenuation at $B$ would be higher, making the absolute attenuation curve steeper in the optical range (but, as it happens, also in the UV; Cardelli et al. 1988). Conversely, two curves that have the same slope in absolute formulation (i.e., they are the same curve, since the value of the absolute curve at $V$ is always 1) will not correspond to the same curves in total formulation unless their $R_{V}$ values are the same.

\subsection{Parameterization of Dust Attenuation Curves}

With just the broadband photometry, one cannot derive the attenuation curve in detail, which is why it is useful to parameterize it. Hence, we follow the methodology of Noll et al. (2009), implemented in CIGALE, whereby the attenuation curve is defined as a two-parameter modification of the total formulation of the Calzetti curve given in Equation (4) of Calzetti et al. (2000). ${ }^{5}$ The exact implementation differs to some extent from that in Noll et al. (2009), so we provide an updated overview here.

The first modification consists of allowing the slope of the curve to deviate from the slope of the Calzetti curve. This is achieved by multiplying the Calzetti curve $\left(k_{\lambda, \mathrm{Cal}}\right)$ with a power-law term having an exponent $\delta$, "centered" at the $V$ band. Negative values of the slope deviation $\delta$ produce attenuation curves that are steeper in $A_{\lambda} / A_{V}$ than the Calzetti curve at $\lambda<5500 \AA$ (Figure 2). The Calzetti curve has $\delta=0$ by definition.

The second modification consists of adding a UV bump, following a Drude profile $D_{\lambda}$ (Fitzpatrick \& Massa 1986) given in Equation (9). ${ }^{6}$ The strength of the bump is specified by the amplitude $B$ in units of $A_{\text {bump }} / E(B-V)$, i.e., it pertains to the total formulation of the attenuation curve, where $A_{\text {bump }}$ is the extra attenuation at $2175 \AA$ due to the bump. We keep the central wavelength and the width of the bump fixed at default CIGALE values of $2175 \AA$ and $350 \AA$, respectively. The Cardelli et al. (1989) MW curve has a bump with a value of $B_{\mathrm{MW}}=3$. It needs to be pointed out that since $B$ is defined in total curve formulation, it does not automatically give the level of contribution of the attenuation due to the bump $\left(A_{\text {bump }}\right)$ to the total attenuation at $2175 \AA\left(A_{2175}\right)$. However, it happens that modified Calzetti curves have similar $k_{2175}=A_{2175} / E(B-V)$ regardless of $\delta$, which implies that $A_{\text {bump }} / A_{2175} \propto B$. Furthermore, since the Calzetti

\footnotetext{
5 For the FUV region $(912-1500 \AA)$, the Calzetti curve (defined at $\lambda>1200 \AA$ ) is substituted with the curve from Leitherer et al. (2002). For simplicity, we will refer to this composite as the just the "Calzetti curve."

6 The Noll et al. (2009) bump profile is equivalent to the one given by Fitzpatrick \& Massa (1986), but the first uses $\gamma$ to denote the width in units of wavelength, while in the other $\gamma$ denotes the width in units of inverse wavelength.
}

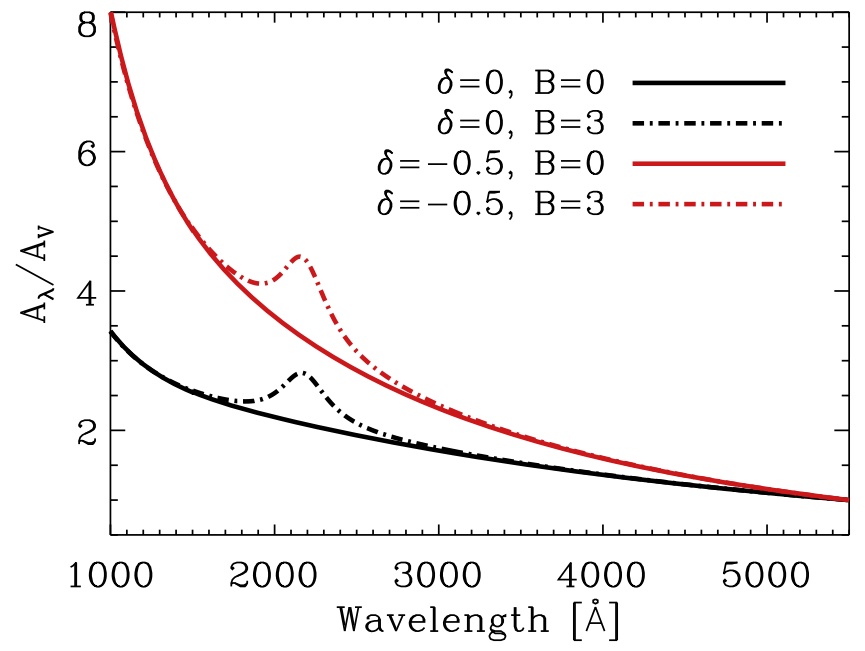

Figure 2. Parameterization of the dust attenuation curves. Following Noll et al. (2009), we define the dust attenuation curve as a two-parameter modification of the Calzetti curve (black solid line), shown in absolute formulation $\left(A_{\lambda} / A_{V}\right)$. The parameter $\delta$ modifies the power-law slope of the curve (with negative values making it steeper in the UV/optical region), while $B$ specifies the amplitude of the $2175 \AA$ bump in the total formulation of the curve $\left(A_{\text {bump }} / E(B-V)\right)$. The fixed $B$ corresponds to a roughly similar level of contribution of the attenuation due to the bump ( $\left.A_{\text {bump }}\right)$ to the total attenuation at $2175 \AA\left(A_{2175}\right)$.

curve and the MW extinction curve have similar slopes in absolute formulation $\left(A_{\lambda} / A_{V}\right)$ but different values of $R_{V}$, it follows that for the same value of $B_{\mathrm{MW}}=3$, the contribution of such bump to the $2175 \AA$ attenuation in the Calzetti curve will be $R_{V, \mathrm{MW}} / R_{V, \mathrm{Cal}} \sim 3 / 4$ of the contribution in the MW curve.

To summarize, the modified Calzetti attenuation law is given as

$$
k_{\lambda, \text { mod }}=k_{\lambda, \mathrm{Cal}} \frac{R_{V, \text { mod }}}{R_{V, \mathrm{Cal}}}\left(\frac{\lambda}{5500 \AA}\right)^{\delta}+D_{\lambda},
$$

where $R_{V \text {,mod }}$ is the ratio of total to selective extinction for the modified law, and is thus dependent on $\delta$ while $R_{V, \mathrm{Cal}}=4.05$. The relationship between $R_{V \text {, mod }}$ and $\delta$ can be derived by imposing $E(B-V)=1$ :

$$
R_{V, \text { mod }}=\frac{R_{V, \mathrm{Cal}}}{\left(R_{V, \mathrm{Cal}}+1\right)(4400 / 5500)^{\delta}-R_{V, \mathrm{Cal}}} .
$$

For example, for $\delta=-0.5, R_{V, \bmod }=2.54$.

We emphasize that in this work we will be deriving attenuation curves that affect individual (young and old) stellar populations. Such curves are more physically relevant than the effective attenuation curve. The distinction arises from the fact that very young stars $(t<10 \mathrm{Myr})$, still embedded in their birth clouds, will suffer higher attenuation than older populations, which will only be attenuated by the diffuse ISM (Fanelli et al. 1988; Keel 1993; Calzetti et al. 1994; Charlot \& Fall 2000; Wild et al. 2011). In order to derive the intrinsic curve, we assume that the attenuation curves for young and old populations

\footnotetext{
The implementation of the modified Calzetti law in CIGALE v0.11 and previous versions of the code adds the bump before applying the power-law modification. However, doing so effectively lowers the bump strength for a given input amplitude, leading to the derived values of $B$ becoming inflated by a factor $R_{V, \mathrm{Cal}} / R_{V \text {,mod. }}$. Our modification of the code implements the bump according to Equation (3). This new method will be the default in CIGALE v0.12 and later.
} 
of a given galaxy are the same $\left(A_{\lambda, \text { young }} / A_{V \text {,young }}=A_{\lambda, \text { old }} / A_{V \text {,old }}\right)$ but have different levels of attenuation. Specifically, we use $E(B-V)_{\text {old }}=0.44 E(B-V)_{\text {young }}$, which is equivalent to $A_{V \text {,young }} / A_{V \text {,old }}=2.27$, following the value for the relationship between stellar and nebular reddening in Calzetti et al. (2000). Assuming a factor of two greater (20 Myr) or smaller (5 Myr) age demarcation between young and old populations does not affect the results significantly. Note that even if both the young and the old populations had the same intrinsic attenuation curves, as assumed in this study, the resulting effective curve will be slightly steeper than these intrinsic curves (by, on average, $\propto \lambda^{-0.2}$ ), because a more highly attenuated young population dominates at shorter wavelengths, making the effective curve steeper. We have verified that assuming, instead, a fixed law for the young population and a variable slope for the older one does not change, on average, the derived slopes of the dust attenuation curve.

In this work, we utilize the fine-resolution model grid specifying SF histories, metallicities, and dust attenuations as in GSWLC-1, while expanding the range and refining the resolution of the two parameters specifying the dust attenuation curve. Specifically, we cover UV bump amplitudes $(B)$ from -2 to 6 , in steps of 2 , and power-law slope deviations $(\delta)$ with exponents from -1.2 to 0.4 , in steps of 0.2 . The range and steps were determined based on the range and accuracy of these parameters in test and mock runs (Appendix A.1). An unphysical negative value for the amplitude of the UV bump was introduced to allow the mean of the relatively wide probability distribution function to assume a value close to zero (see a similar discussion in Salmon et al. 2016), and thus obtain an unbiased result for galaxies that in reality lack the UV bump. Keeping the high grid resolution as in GSWLC-1, while treating the dust attenuation curve parameters as essentially free, results in 2.8 million distinct models at each redshift. Models are calculated at 30 redshifts $(0.01<z<0.30)$, separated by 0.01 .

We refer to the physical parameters determined from this new, SED+LIR fitting as GSWLC-2 (the medium-deep catalog would be referred to as GSWLC-M2), and will make it publicly available on the project Web site. ${ }^{8}$

\section{Results}

In this section, we first explore the demographics of dust attenuation curves ("curves") of different populations of galaxies and then present average curves in relation to curves from prior work, together with analytical expressions for their construction.

We characterize the curves by the parameters $\delta$ and $B$, representing the power-law slope deviation with respect to the Calzetti curve and the strength of the UV bump in the total $(k)$ formulation of the attenuation curve. Values $\delta<0$ signify curves that are steeper than the Calzetti curve in the UV and blue optical. For reference, the MW bump in the extinction curve has a mean strength of $B=3$. However, the contribution of the bump with amplitude $B$ to the attenuation at $2175 \AA$ will be smaller $(\sim 3 / 4)$ for the modified Calzetti curve than for the MW extinction curve.

For the sample of star-forming galaxies, which will be our main focus, the mean error of the dust attenuation curve slope deviation, calculated as the standard deviation of the

\footnotetext{
http://pages.iu.edu/ salims/gswlc
}

probability distribution function, is 0.25 , with the 90th percentile range of errors extending from 0.1 to 0.4 . The mean error of the determination of the bump amplitude is 1.8, with the 90th percentile range between 0.5 and 2.7. Mock SED fitting (Appendix A.1) shows that despite the dispersion, both the slope and the bump strength are recovered without significant systematics. In what follows, we will focus on the average curve properties of a binned distribution of galaxies, which are robustly measured due to the large number of galaxies in the sample.

\subsection{Trends between Dust Attenuation Curve Parameters and Galaxy Physical Parameters}

In this section, we present average attenuation curve parameters for galaxies of different stellar masses, as a function of various physical and geometric parameters.

Figure 3 shows contour maps of average dust attenuation curve parameters on sSFR $-M_{*}$ diagrams. Diagrams of sSFRs versus the stellar mass have emerged as an essential tool for the characterization of galaxy populations and their evolution (Guzmán et al. 1997; Pérez-González et al. 2003; Bauer et al. 2005; Salim et al. 2007). Diagrams featuring the SFR normalized by the stellar mass (sSFR) are easier to interpret than the equivalent $\mathrm{SFR}-M_{*}$ diagrams and are more fundamental than the related color-magnitude diagrams. The upper panels of Figure 3 show the full sample, consisting of both actively star-forming (typically $\log$ sSFR $>-11$ ) and quiescent galaxies, while the lower panels show only BPT-classified star-forming galaxies. The white line represents the median sSFR trend of star-forming galaxies (the star-forming sequence) and is repeated in the upper panels.

Focusing on the left two panels of Figure 3, we see that the average slopes of the attenuation curves span a significant range of values across the sSFR $-M_{*}$ plane. Attenuation curves are on average the shallowest (lightest shade) for the most massive star-forming galaxies, having slopes around that of the Calzetti curve $(\delta=0)$. However, both the less massive starforming galaxies as well as the more quiescent galaxies have significantly steeper curves, up to $\delta \sim-0.9$, which is steeper than the slope of SMC extinction curve (roughly $\delta=-0.45$ ). There are no regions with average slopes shallower than the Calzetti curve.

For star-forming galaxies (lower-left panel), the primary trend in the slope is the one with respect to the mass (higher mass = shallower slope), but there is also a secondary trend with sSFR: at any given mass, the curves tend to be steeper on both sides of the main sequence (the shallowest slopes at a given mass are slightly below the main sequence). In particular, a galaxy with a high (s)SFR for its mass (a "starburst") will have a somewhat steeper curve than the galaxy of the same mass and with a more typical (s)SFR (lying closer to the white line). We will show shortly that the trend with mass is a consequence of the more fundamental dependence on optical opacity, while the trend with sSFR is independent from it. Starforming galaxies, like the general population, on average have steep slopes (Section 4.2).

We now focus on the right panels of Figure 3, showing the amplitude of the UV bump in bins of sSFR- $M_{*}$ parameter space. Again there is a range of values, from zero to MW-like. The bump is most pronounced in star-forming galaxies of lower mass and for quiescent galaxies. Massive star-forming galaxies $\left(\log M_{*} \sim 11\right)$ are consistent with having little or no bump. This suggests either 

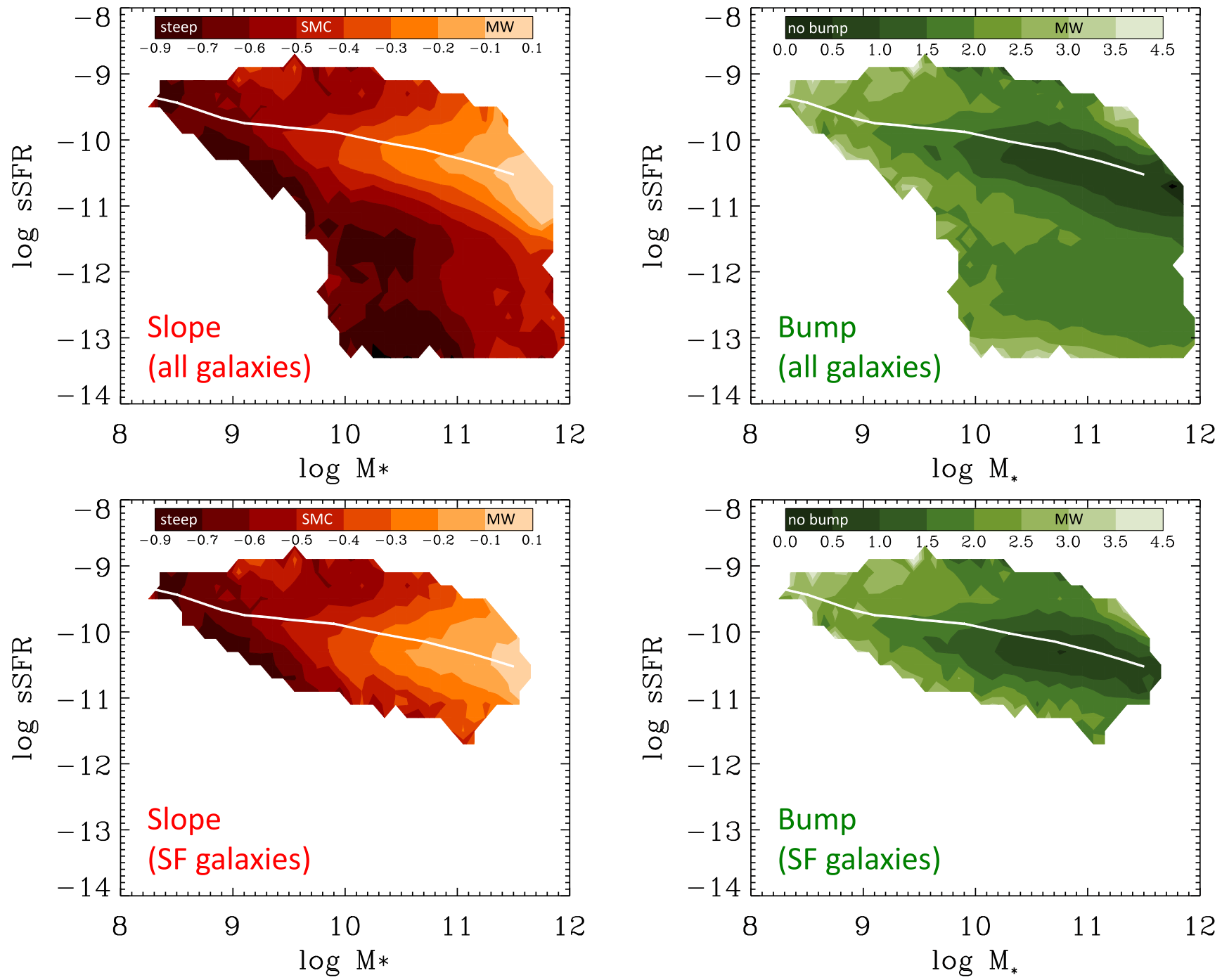

Figure 3. Contour maps of the dust attenuation curve parameters (average slope (left panels) and average UV bump strength (right panels)) as a function of the specific SFR and stellar mass, for all galaxies (upper panels) and galaxies classified as star-forming (lower panels). The white line represents the median sSFR for star-forming galaxies (the "main sequence" of SF). The slope is expressed as the exponent of the power-law deviation $(\delta)$ with respect to the Calzetti curve, the latter being represented by the lightest contour $(\delta=0)$. There is a wide range of curve slopes, with lower-mass galaxies having steeper slopes. Bump strengths (amplitudes in units of $A_{\text {bump }} / E(B-V)$, which for the Milky Way extinction curve has a typical value of 3) are on average weaker than the MW extinction curve bump, especially for star-forming galaxies of higher mass. Bins with 10 or more galaxies are shown.

that the MW is atypical for a galaxy of its mass or that its extinction curve does not reflect its attenuation curve if it were to be observed as an external galaxy. The LMC, which also has an MW-like bump agrees better with other galaxies of that mass. No group of galaxies has bumps significantly stronger than the one in the MW extinction curve. As in the case of the slope, the strength of the bump exhibits a correlation with the sSFR, increasing on both sides of the main sequence. Unlike the slope, the trends of bump strength against the mass or sSFR are not the result of some more fundamental trend with the level of attenuation, either in $V$ or in FUV. We conclude that, on average, the UV bump in galaxies is moderate compared to the MW bump.

In the rest of the paper, we focus on galaxies classified as star-forming, for which the dust attenuation parameters are derived with greater precision and which are observed at a wide range of redshifts.
Next we explore, again in the form of contour maps, the dependence of curve parameters on the level of attenuation in the optical $(V)$ and the FUV. These attenuations are effective, arising from both the young and old populations. The left panels of Figure 4 show slope deviations as a function of $A_{V}$ and $M_{*}$ in the upper panel, and $A_{\mathrm{FUV}}$ and $M_{*}$ in the lower panel. The white line shows the median attenuation for galaxies of different masses. While galaxies tend to have higher attenuation as the mass increases, there is a substantial scatter, especially for galaxies of higher mass. The slope of the attenuation curve is strongly correlated with optical attenuation, with galaxies with low $A_{V}$ values having steep curves and the ones with high $A_{V}$ being shallow. Since the more massive starforming galaxies have higher optical opacities (the rising white line in the upper-left panel of Figure 4), the trend between the slope and a mass seen in Figure 3 is simply the result of the 

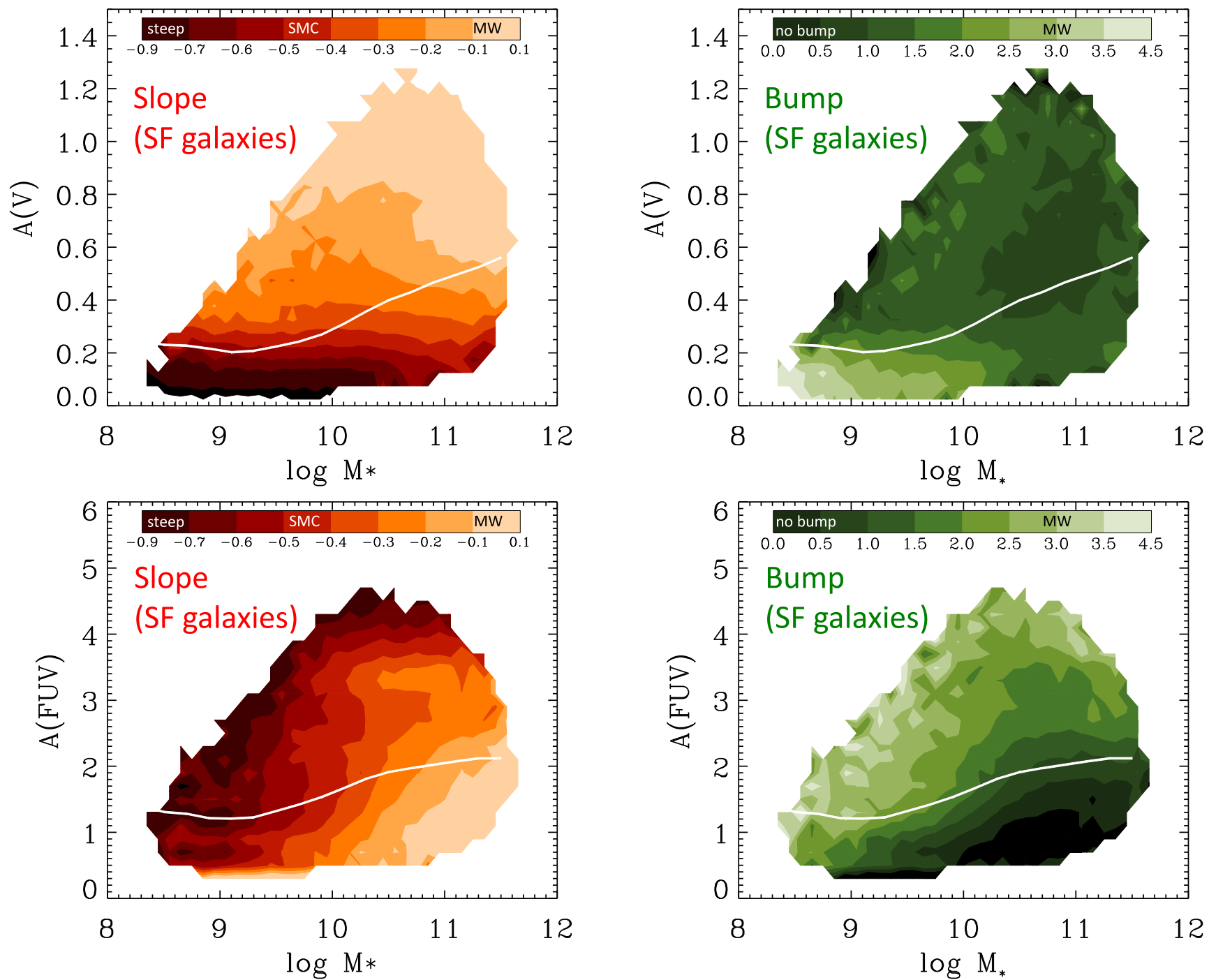

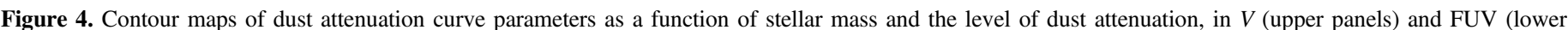

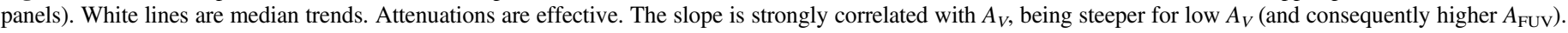
The UV bump is stronger for galaxies with lower optical opacity.

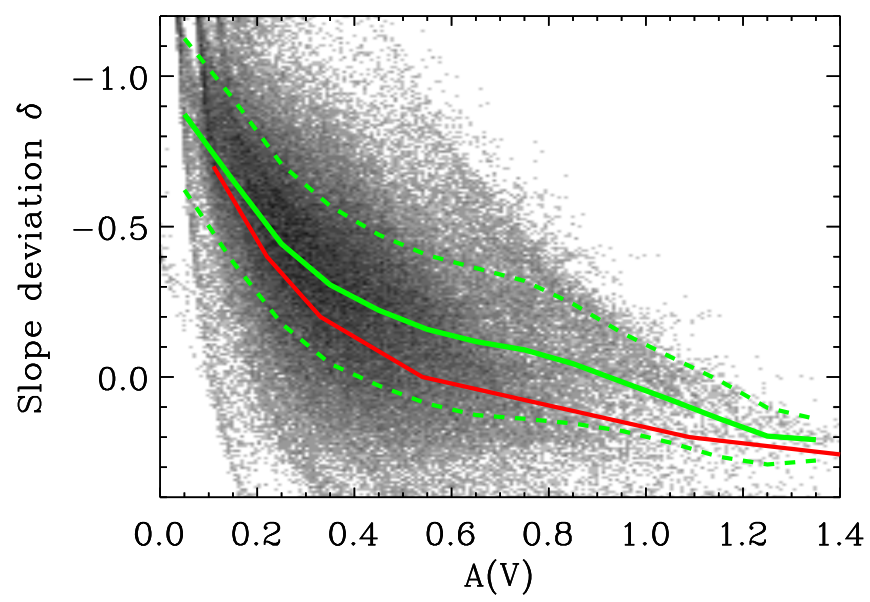

Figure 5. Correlation between the dust attenuation slope and effective optical opacity. The attenuation curves of galaxies with higher optical opacity are systematically shallower. The slope is expressed as the exponent of the powerlaw deviation $(\delta)$ with respect to the Calzetti curve $(\delta=0)$. The average trend and the $1 \sigma$ range around it are shown as green curves. The relation is in qualitative agreement with the Chevallard et al. (2013) modelling results (red curve). A mild discretization of model grid parameters is visible. underlying trend between the slope and $A_{V}$. At fixed $A_{V}$ there is no trend of slope versus mass (contours are horizontal in Figure 4, upper left).

The trend between slope deviation and $A_{V}$ is shown separately in Figure 5. The correlation is strong, and the scatter at a given $A_{V}$ is comparable to the slope determination error. In other words, there may be little intrinsic scatter. From this, we see that only the galaxies with substantial optical attenuation $\left(A_{V}>0.8\right)$ tend to have, on average, curves as shallow as the Calzetti curve. The dependence of the slope on the FUV attenuation is to some degree reversed: the slopes are steeper as the attenuation increases or as the mass decreases. The opposite sense of the trends may appear paradoxical: one expects dust attenuation in the UV to follow the attenuation in the optical. This would have been the case if galaxies obeyed a universal attenuation law, but is not necessarily true when there is a wide range of dust attenuation slopes, as is the case here. This can be seen in Figure 6 , where $A_{\mathrm{FUV}}$ is plotted against $A_{V}$. The upper envelope of points corresponds to the steepest slopes, whereas the lower envelope corresponds to the shallowest slopes. 


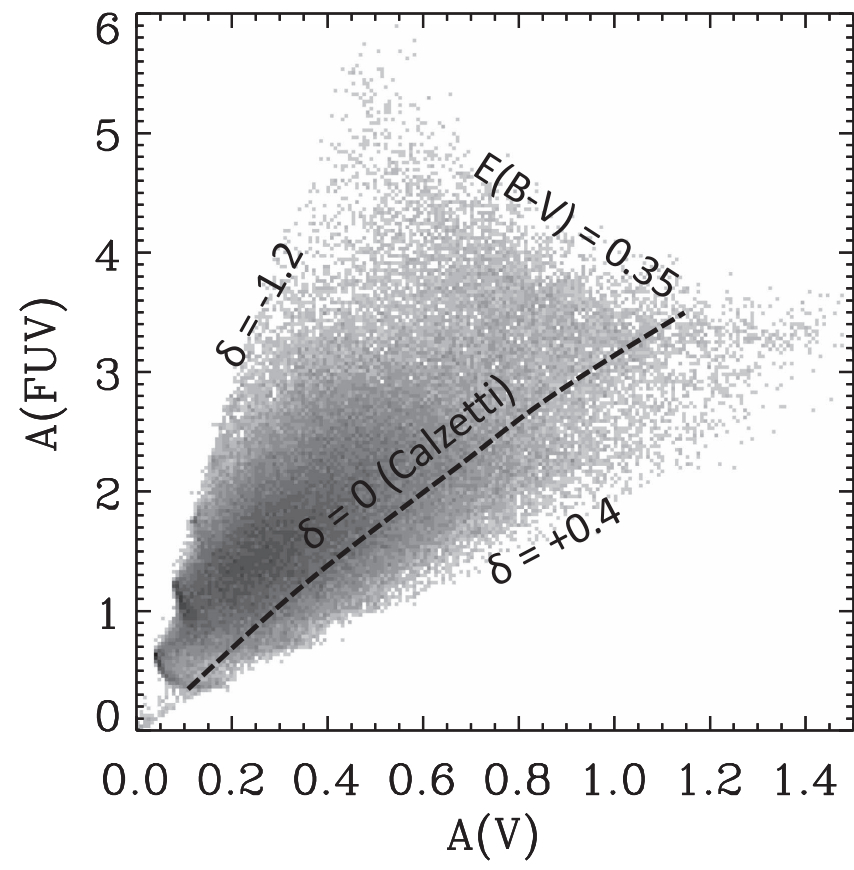

Figure 6. Effective attenuation in FUV vs. the attenuation in $V$. Because of the wide range of slopes of dust attenuation curves, the distribution is very wide. The upper and lower envelopes correspond to extrema in slopes. The locus of points for the Calzetti curve is indicated. The upper-right envelope is defined by the galaxies with the highest reddening (labeled). Galaxies with similar $E(B-V)$ run parallel to this label.

The UV bump, as mentioned, tends to be more prominent in galaxies with steeper slopes, which, on average means lower $A_{V}$ and higher $A_{\mathrm{FUV}}$. This agrees with the trends in the right panels of Figure 4 and has been detected even at higher redshifts (Kriek \& Conroy 2013; Tress et al. 2018). An apparent correlation between the bump intensity and slopes may raise the concern that the steep curves that we are finding are an artifact of some underlying degeneracy with the bump. To test for this possibility, we produce a run in which the bump is not allowed, whereas all the other parameters of the SED fitting are identical. If the slope and the bump are artificially correlated, not allowing for the bump would force the slope to be systematically different from the nominal slope. Figure 7 compares the slopes from the two runs. There is no systematic difference: the slopes remain the same on average regardless of whether or not the bump was allowed. We conclude that the bump is correlated, but is not confounded, with the slope.

A related concern is that nonzero bump amplitudes are just fitting artifacts. We have performed a test to check if this might be the case. We perform the fitting without the NUV band, using the models without the UV bump. We then look at the difference in the NUV magnitude predicted by the no-bump SED fitting and the actual NUV magnitude. We find that the predicted no-bump magnitude is on average brighter than the actual magnitude for galaxies with higher sSFRs, in accordance with the bump amplitude derived from the SED fitting. However, it should be pointed out that the effect on NUV magnitude, even of an MW-like bump, is small (0.2 mag), and in the case of our average bumps, it is less than 0.1 mag.

In Figure 8, we look at the trends of the attenuation curve parameter versus gas-phase metallicity (upper panels) and inclination (lower panels). The metallicity has been calculated

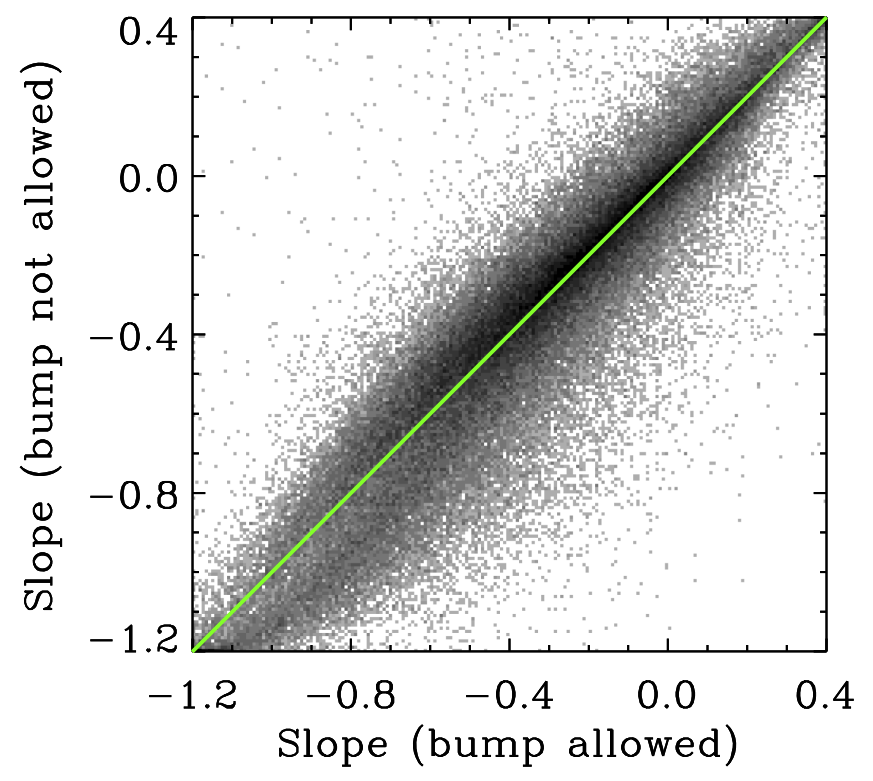

Figure 7. Comparison of dust attenuation slopes (i.e., their deviations from the Calzetti curve) for the nominal SED-fitting run, and one in which the bump is not allowed. The slopes show no systematic offset (the green line is the 1:1 line), indicating that their steepness is not an artifact of allowing for a potentially poorly constrained bump.

using the N2O2 method (the ratio of [N II]6584 to [O II]3727) and calibration from Kewley \& Dopita (2002). This is the method of choice because it is less sensitive to the ionization parameter than most other commonly used methods (Kewley \& Dopita 2002). For both the slope and the bump strength, the contours are mostly vertical, meaning that there is not much dependence on the metallicity.

The trends involving galaxy inclination (derived as the simple axis ratio from the exponential fit to the galaxy's 2D profile; $A B_{-}$exp) are shown in the lower panels of Figure 8. The bump is largely insensitive to the galaxy orientation. There is some trend for the slope of nondwarf (presumably more disky) star-forming galaxies, in the sense that face-on galaxies have slightly steeper slopes. This trend is not independent of the trend on optical opacity and largely goes away when $A_{V}$ is controlled for.

\subsection{Average Dust Attenuation Curve: Comparison with Literature Curves}

In this section, we present average dust attenuation curves for all star-forming galaxies, for star-forming galaxies binned by stellar mass, and for high-redshift analogs (starbursts). For each galaxy in these groups, we reconstruct individual $A_{\lambda} / A_{V}$ curves out of $\delta$ and $B$ values, and then average the curves in small wavelengths bins from $912 \AA$ to $2.2 \mu \mathrm{m}$. We additionally characterize these curves by the overall power-law exponent $n$ (following Charlot \& Fall 2000) determined from a fit (in the $\log$ of $\lambda$ ) to the curve in the range of $912 \AA$ to $2.2 \mu \mathrm{m}$ :

$$
A_{\lambda} / A_{V}=(\lambda / 0.55 \mu \mathrm{m})^{-n}
$$

Our average curve for star-forming galaxies is shown in Figure 9 as a black curve, with the gray region showing $1 \sigma$ dispersion across the sample. Dispersion is zero at $5500 \AA$ by construction. We see that the average curve $(n=1.15)$ is almost as steep as the SMC curve (Prevot et al. 1984; yellow 

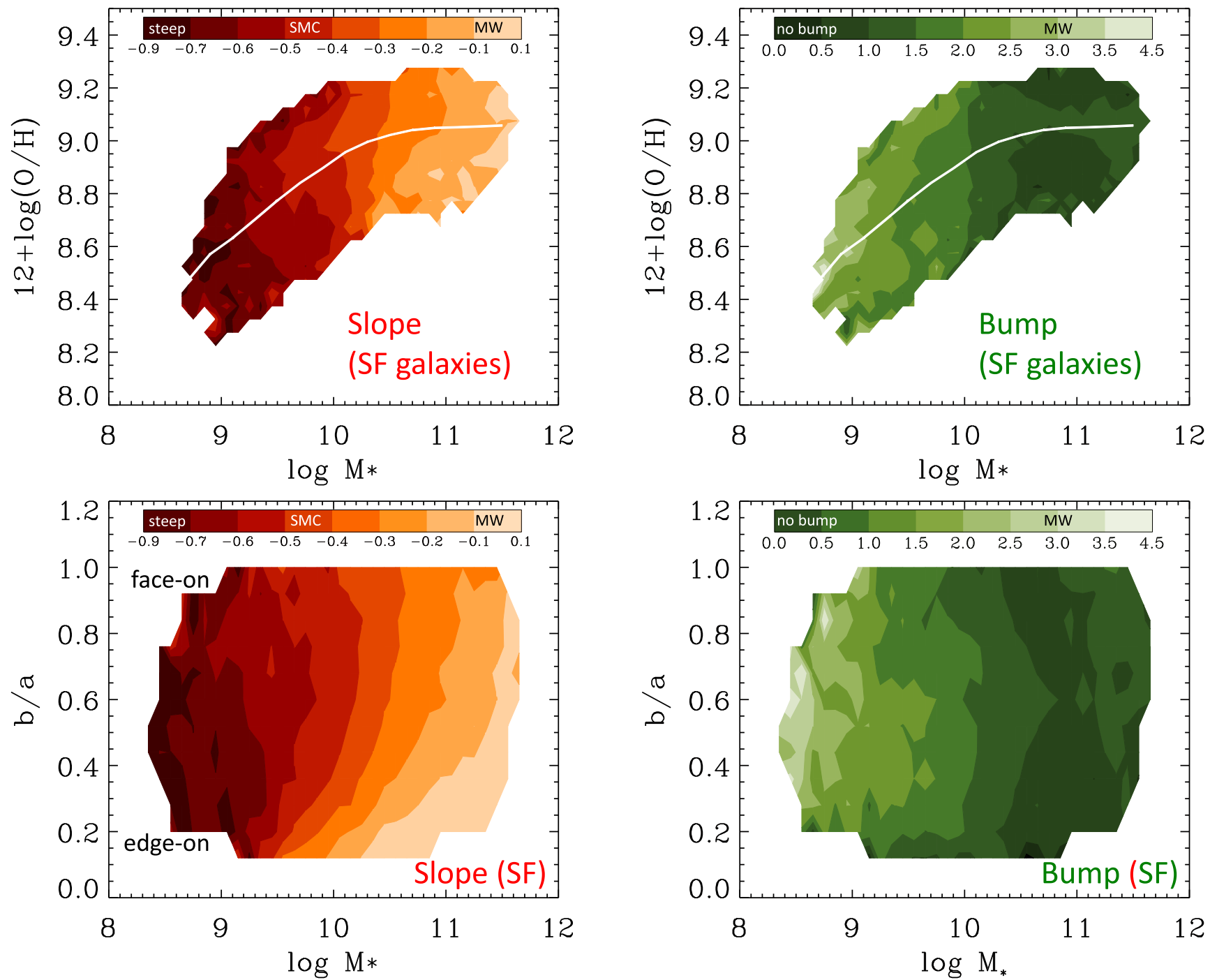

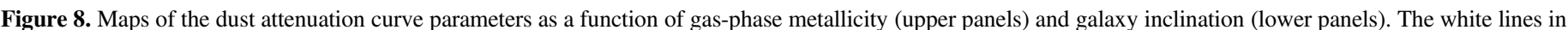

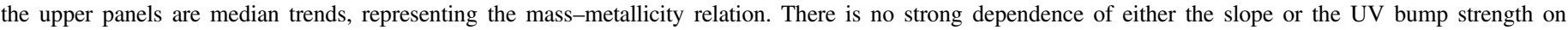
metallicity, while the slope is shallower for more inclined massive galaxies.

curve, $n=1.2$ ). The average curve exhibits a moderate UV bump, whose contribution to attenuation at $2175 \AA$ is roughly one-third that of the contribution of the MW bump to its curve at $2175 \AA$. The effect of such a bump on the observed NUV magnitudes is $\sim 0.07 \mathrm{mag}$. The $\mathrm{MW}$ extinction curve from Cardelli et al. (1989; red curve) is less steep overall ( $n=0.9)$, but soars at the FUV and has a more pronounced UV bump. Given that MW and SMC are extinction, not attenuation, curves, they are of limited value for drawing conclusions as to how well the MW or the SMC follows our mean curve. The Calzetti curve is similar to the MW curve except that it is shallower in the FUV and does not exhibit the bump, with an overall power-law slope of $n=0.75$. The range of curves found in this work extends from Calzetti-like curves at the shallow extreme, up to the steeper than SMC curves $(n=1.6)$ on the other end. We also show a range of $A_{\mathrm{FUV}} / A_{V}$ values corresponding to SDSS/GALEX galaxies fitted to a suite of model SEDs based on Charlot \& Fall (2000). Charlot \& Fall (2000) models, having different $A_{V}$ normalizations for young and old populations and the intrinsic slope of $n=0.7$, naturally produce a range of effective attenuation curve slopes, which is, however, still smaller and on average less steep $(n=0.9)$ than what we find for the same galaxies (cf. Hayward \& Smith 2015).

In Figure 10, we show the average curves for star-forming galaxies, now split into three bins of stellar mass (black curves). The curve for the massive galaxies $\left(\log M_{*} \sim 11\right)$ is the shallowest $(n=1.0)$, but still not as shallow as the Calzetti curve. The average curve for less massive galaxies is steeper still, with a moderate UV bump. Conroy et al. (2010) have used UV (GALEX) and optical (SDSS) colors to constrain the dust attenuation curve of a $9.5<\log M_{*}<10$ sample and have arrived at the values shown in Figure 10 as green squares. There is a good overall agreement with our results for galaxies of comparable mass. We can derive the expression between the average slope deviation and the mass as

$$
\delta=-0.38+0.29\left(\log M_{*}-10\right),
$$

from which the overall power-law slope can be obtained as $n=-\delta+n_{\text {Cal }}=-\delta+0.75$.

We also derive an average curve for high-redshift analogs. Following Salim et al. (2015), we define high-redshift analogs as galaxies with a large positive offset from the main sequence, 


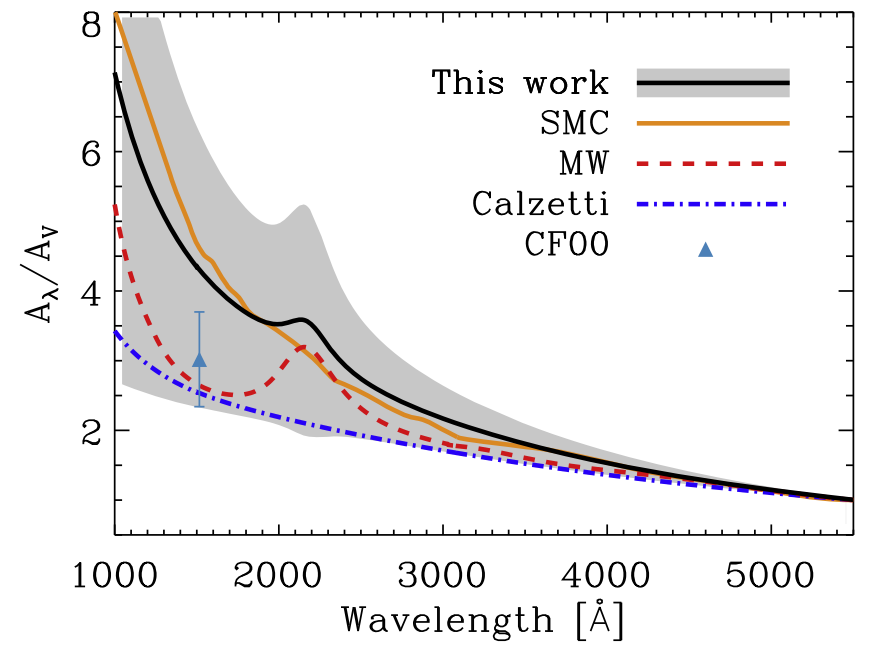

Figure 9. Comparison of our average dust attenuation curve for star-forming galaxies (black line) to several canonical attenuation and extinction curves. Curves are normalized to $A_{V}$. Our average curve is steeper than the MW (Cardelli et al. 1989; O'Donnell 1994; red dashed line) and Calzetti et al (2000) curves (blue dotted-dashed) and is more similar in slope to the SMC curve (Prevot et al. 1984; orange). The UV bump is one-third the Milky Way bump. Our curves for the individual galaxies span quite a range- the gray area shows the $1 \sigma$ dispersion around the mean curve. The triangle shows the average and the $1 \sigma$ range of the curves obtained by applying the Charlot \& Fall (2000) model with the intrinsic slope of $n=0.7$.

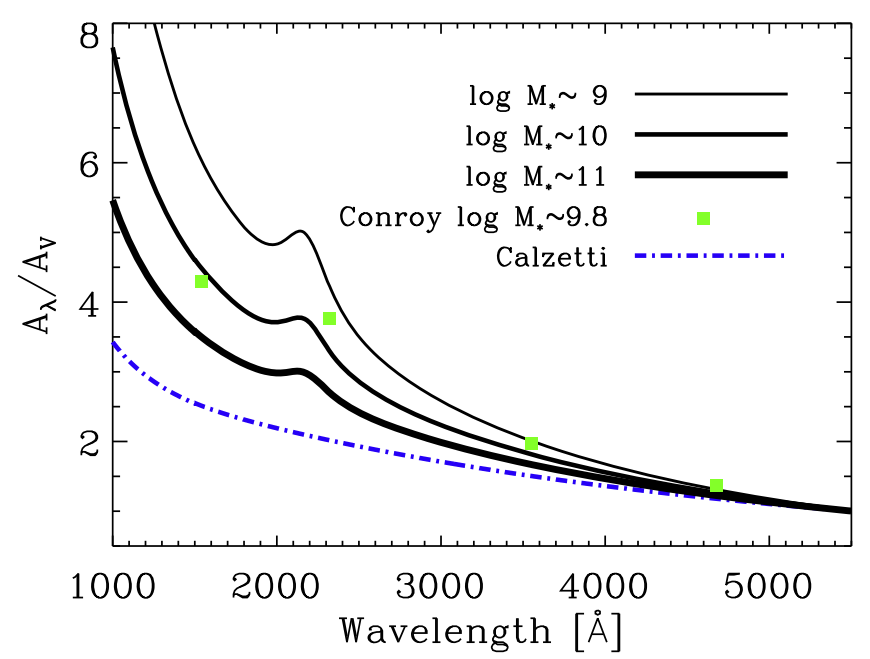

Figure 10. Comparison of our average dust attenuation curves for galaxies of different mass (black lines) with several curves from the literature. Curves of lower-mass galaxies tend to be steeper, which we find to be correlated with their lower dust content. There is a good agreement with the points (green squares) corresponding to the attenuation curve of Conroy et al. (2010), derived from UV-optical photometry of a sample of $9.5<\log M_{*}<10$. The relative contribution of the UV bump declines with mass.

specifically, $\Delta \operatorname{logSSFR}>0.5$ (the results are the same if a more extreme criterion of at least 1 dex of offset is adopted.) It is worth pointing out that selecting high-redshift analogs (or, more generally, starbursts) based on the offset from the main sequence is in close accordance with how galaxies evolve (the shifting normalization of the main sequence). Using instead some cutoff in the sSFR would preferably select low-mass galaxies (Figure 3), whereas a cut in the SFR would preferably select high-mass galaxies. The sample of Calzetti et al. (1994)

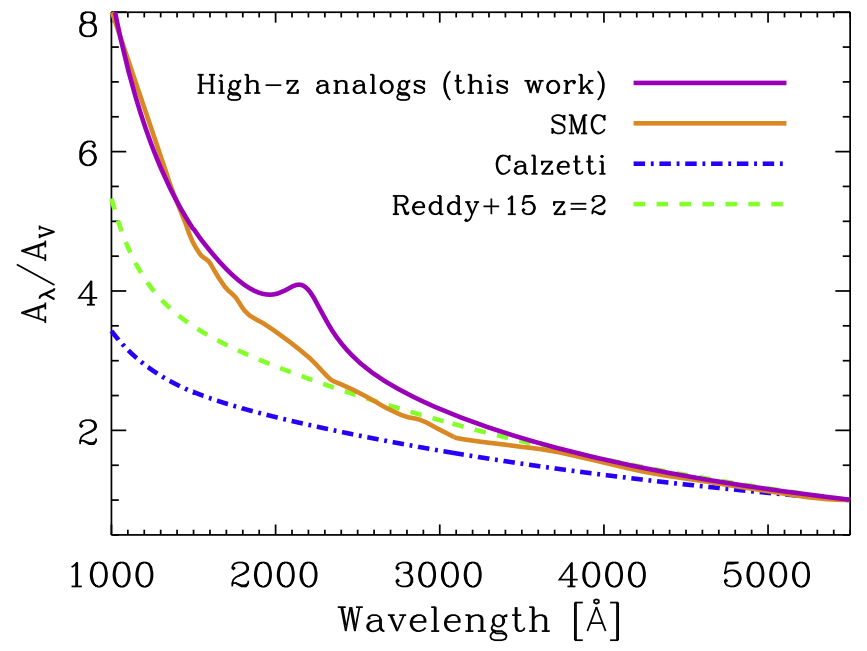

Figure 11. Comparison of average dust attenuation curves for our sample of high-redshift analogs (galaxies lying $>0.5$ dex above the local main sequence; purple line) and several curves from the literature, including Reddy et al. (2015; green dashed curve). Local analogs (starbursts) have steep curves, similar to the SMC slope (orange; Prevot et al. 1984), but with more extinction in the NUV, partly due to the moderate UV bump. From this we argue that high-redshift galaxies have a range of curves that are on average as steep as or even steeper than the local galaxies and therefore as steep as or steeper than the SMC curve.

lies, on average, 1 dex above the local main sequence, i.e., it occupies a similar position as our $\Delta \operatorname{logSSFR}>0.5$ selection.

The resulting curve for high-redshift analogs is shown in Figure 11 in purple. It is similar to the curve for the general population of intermediate-mass galaxies, with an SMC-like FUV rise $(n=1.25)$, but a somewhat higher attenuation in the NUV (partly due to a moderate bump). The curve of Reddy et al. (2015) derived from $z \sim 2$ galaxies using the methodology of Calzetti et al. $(1994,2000)$ is less steep than ours (green dashed curve). Their selective curve is essentially identical to the Calzetti curve in the UV/optical region, but is effectively steeper in absolute normalization because of the lower $R_{V}$ (2.505 versus 4.05). Our steep curve for high- $z$ analogs agrees better with a recent reassessment of $z \sim 2$ observations by Reddy et al. (2018), who find that the SMC-like curve gives a better match to the observed IRX $-\beta$ relation than the shallower curves. What is significant is that we find that such a steep curve is needed for local starbursts as well, and that such galaxies have a slightly steeper curve, on average, than the general population of local star-forming galaxies. We discuss this result further in Section 5. A correlation between the slope deviation and the stellar mass is present for high-redshift analogs as well, and is given by

$$
\delta=-0.45+0.19\left(\log M_{*}-10\right) .
$$

\subsection{Functional Fits}

We fit our curves with polynomial functions following the formalism of Calzetti et al. (2000), where the fit is performed in the total formulation of the curve, from which an absolute curve can obtained by dividing by $R_{V}$ (Section 3.3). Calzetti et al. (2000) present their curve as a piecewise fit: a third-order polynomial in $\lambda^{-1}$ for the UV+blue optical region, and a linear function in $\lambda^{-1}$ for the red optical+near-IR region, with the split between two regimes at $0.63 \mu \mathrm{m}$. Considering that the level of uncertainty far exceeds the refinement provided by 
Table 1

Functional Fits of Dust Attenuation Curves

\begin{tabular}{lccccccc}
\hline \hline Sample & $R_{V}$ & $B$ & $a_{0}$ & $a_{1}$ & $a_{2}$ & $a_{3}$ & $\lambda_{\max }(k>0)$ \\
\hline Star-forming galaxies & 3.15 & 1.57 & -4.30 & 2.71 & -0.191 & 0.0121 & 2.28 \\
$\quad 8.5<\log M_{*} \leqslant 9.5$ & 2.61 & 2.62 & -3.66 & 2.13 & -0.043 & 0.0086 & 2.01 \\
$9.5<\log M_{*} \leqslant 10.5$ & 2.99 & 1.73 & -4.13 & 2.56 & -0.153 & 0.0105 & 2.18 \\
$10.5<\log M_{*} \leqslant 11.5$ & 3.47 & 1.09 & -4.66 & 3.03 & -0.271 & 0.0147 & 1.43 \\
High- $z$ analogs & 2.88 & 2.27 & -4.01 & 2.46 & -0.128 & 0.0098 & 1.20 \\
$\log M_{*} \leqslant 10$ & 2.72 & 2.74 & -3.80 & 2.25 & -0.073 & 0.0092 & 2.12 \\
$\log M_{*}>10$ & 2.93 & 2.11 & -4.12 & 2.56 & -0.152 & 0.0104 & 2.05 \\
Quiescent galaxies & 2.61 & 2.21 & -3.72 & 2.20 & -0.062 & 0.0080 & 1.00 \\
\hline
\end{tabular}

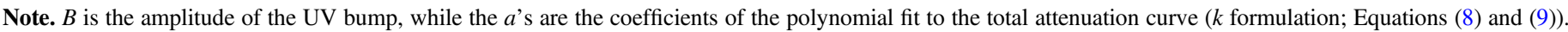

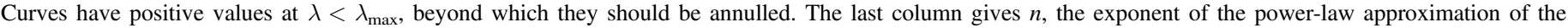

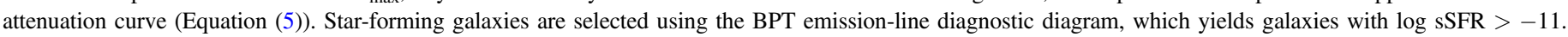

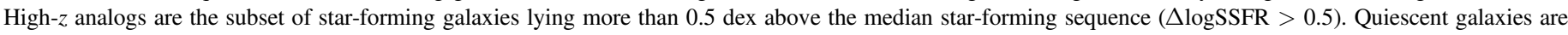
galaxies with $\log \mathrm{sSFR}<-11$. Star-forming galaxies and high- $z$ analogs are additionally split by stellar mass.

splitting the curve into two parts, we derive functional fits based on the entire $0.912<\lambda<2.2 \mu \mathrm{m}$ range using the thirdorder polynomial

$$
k_{\lambda}=a_{0}+a_{1} \lambda^{-1}+a_{2} \lambda^{-2}+a_{3} \lambda^{-3}+D_{\lambda}(B)+R_{V}
$$

to which we add the UV bump as a Drude profile $D_{\lambda}(B)$, with amplitude $B$ and fixed central wavelength $(2175 \AA)$ and width $(350 \AA)$ :

$$
D_{\lambda}(B)=\frac{B \lambda^{2}(0.35 \mu \mathrm{m})^{2}}{\left[\lambda^{2}-(0.2175 \mu \mathrm{m})^{2}\right]^{2}+\lambda^{2}(0.35 \mu \mathrm{m})^{2}} .
$$

For the average curve of all star-forming galaxies, we get $R_{v}=3.15$, and the fit

$$
\begin{aligned}
k_{\lambda}= & -4.30+2.71 \lambda^{-1}-0.191 \lambda^{-2}+0.0121 \lambda^{-3} \\
& +D_{\lambda}(1.57)+3.15,0.09 \leqslant \lambda<2.2 \mu \mathrm{m} .
\end{aligned}
$$

Note that the fit produces negative values for $\lambda>2.28 \mu \mathrm{m}$, so it is recommended to replace it with zero values in that regime.

An alternative to using the polynomial fits is to use the expression for the modified Calzetti law (Equations (3) and (4)) with the values $\delta=-0.4$ and $B=1.3$. A somewhat cruder but still useful characterization of the curve is a simple power-law form (Equation (5)). Considering that the UV bump is relatively modest in average curve, ignoring it should not lead to large systematics. Thus, our mean law can be approximated with an absolute curve with the best-fitting power-law exponent of $n=1.15$.

Considering the diversity of dust attenuation curves, it is useful to have functional forms for specific subsamples. Table 1 lists polynomial coefficients for the fits of star-forming galaxies split by stellar mass. Furthermore, we present fit parameters for high-redshift analogs: all and split by mass, and for the quiescent galaxies (logsSFR $<-11$; any BPT class).

It should be pointed out that unless the IR dust emission constraints are used, one should refrain from leaving the curve as a free parameter in the SED fitting, as the resulting illconstrained values may bias the derived values of SFRs. In such cases, it is preferable to use a fixed, but appropriate, average curve.

\section{Discussion}

The main results of our work are, first, that galaxies exhibit a very wide range of attenuation curves, and second, that the slopes are on average steep, both for normal star-forming galaxies, and, to an even greater degree, for galaxies above the main sequence, i.e., starbursts or high- $z$ analogs. We have performed a number of tests (effective versus intrinsic curve, different old/young $A_{V}$ normalizations, different old/young transition timescales, different dust absorption factors for ionizing photons, fitting with and without the bump, different assumed input errors, Chabrier versus Salpeter IMF, (non) correction of emission lines, Maraston (2005) versus Bruzual \& Charlot (2003) SPS models, different SF histories, fitting with and without the FUV), and the need for overall steep curves with moderate bumps remains.

Our results agree with other studies that employ model-based methods, which point to generally steep curves with moderate bumps (less than $B_{\mathrm{MW}}=3$ ) at both low and high redshifts. We review these results, first focusing on low redshift. Conroy et al. (2010), using UV/optical photometry of samples of galaxies at different inclinations, find a steep slope with a bump of $B=2.4$, consistent with what we find for galaxies of similar mass (Figure 10). Burgarella et al. (2005), using UV to far-IR SED fitting, also find on average a steep $(n=1.1)$ and a moderately bumpy slope for a sample of $\sim 100$ local galaxies. Most recently, Leja et al. (2017) used UV-to-mid-IR SED fitting of a sample of 129 nearby galaxies to constrain physical parameters including the slope of the attenuation curve. They use a similar methodology to ours, except that the determination of $L_{\mathrm{IR}}$ is not decoupled from the overall SED fitting. They find the same range of slopes as we do $(-1.2<\delta<0.4)$, but on average somewhat shallower, consistent with the fact that their sample contains, on average, more massive galaxies. We find that the dust attenuation curves tend to be steep also for quiescent galaxies, which lie below the main sequence. Recently, Viaene et al. (2017) obtained an optical attenuation curve of a nearby lenticular, star-forming galaxy with prominent dust lanes, one of very few individual galaxies with a determined attenuation curve. They find a steep curve with a slope deviation of $\delta=-0.43$, in agreement with our results for galaxies lying below the main sequence (Figure 3, upper left).

Our results also agree with the results of studies that apply model-based methods to a general population of higher-redshift 
galaxies. Kriek \& Conroy (2013), using stacked SEDs of medium- and broadband photometry of galaxies at $0.5<z<2$, find an average slope of $\delta=-0.2$ and an average bump of $B=1$, with both parameters spanning a range of values (their Figure 2). Their sample is restricted to galaxies with moderate to high optical attenuations $\left(A_{V}>0.5\right)$, which have shallower slopes and weaker bumps (Figures 4,5 ). When we isolate $A_{V}>0.5$ galaxies, the average values of the curve parameters agree with those found by Kriek \& Conroy (2013). Evidence for a moderate bump $(0.5<B<2.5)$ and a range of steep curves $(-0.7<\delta<0.1)$ has also been found in the SED analysis of a $1<z<2.2$ sample by Buat et al. (2012), as shown in their Figure 8. A similar range of slopes, though on average somewhat shallower than ours, was obtained in the SED analysis of rest-frame UV to optical data of $z \sim 2$ galaxies (Salmon et al. 2016).

The strong correlation between the attenuation curve slope and optical depth (Figure 5) is a result with important implications, and it also impacts the underlying assumption of the comparison method, which we will discuss shortly. Our results match the predictions of Chevallard et al. (2013), which are based on radiative transfer models combined with realistic dust geometries and the two-component (birth clouds/diffuse ISM) model (Charlot \& Fall 2000). In particular, the $A_{V}$ point at which the dependence of slopes on $A_{V}$ changes its character from rapid (at $A_{V}<0.4$ ) to less rapid (at $A_{V}>0.4$ ) matches the break in our trend (Figure 5). The existence of a dependence of the attenuation curve slopes on $A_{V}$ can also be inferred from the SED-fitting results at higher redshift (Figure 12 of Arnouts et al. 2013) and has been reported recently in Leja et al. (2017) for a sample of 129 nearby galaxies.

Chevallard et al. (2013) provide the following physical picture for this correlation. The steepness of an attenuation curve at small optical depths is the result of the dominance of scattering over absorption, coupled with the fact that scattering is more forward directed at shorter wavelengths whereas it is more isotropic at longer wavelengths. As the optical depth increases, absorption becomes more dominant than scattering, and the curve becomes shallower (grayer).

We show that the dependence of the slope of the curve on $A_{V}$ leads to an apparent dependence on the stellar mass. A similar result, that $\log M_{*}<10$ galaxies have a steeper curve than more massive galaxies, was recently obtained in the analysis of a large sample of $z \sim 2$ galaxies by Reddy et al. (2018).

Chevallard et al. (2013) furthermore show that the dependence of the slope on $A_{V}$ is the same irrespective of whether the $A_{V}$ is driven by different levels of intrinsic (face-on) attenuation or is the result of inclined viewing geometry. Regarding the latter, they predict that attenuation slopes should steepen at $b / a>0.4$ (going from edge on to face on), in agreement with our results for disk-geometry galaxies (Figure 8, lower-left panel).

The strength of the UV bump also shows some correlation with $A_{V}$ (Figure 4, upper-right panel). A similar behavior has been recently seen by Hagen et al. (2017) in their analysis of SMC dust curves based on pixel SED fitting of UV (from Swift), optical, and 3.6 and $8 \mu \mathrm{m}$ photometry. Interestingly, this study finds that SMC exhibits a mild UV bump, on average one-fifth as strong as the MW extinction curve bump. Furthermore, they show that the bump amplitude is highest
( $B \sim 1.5$ ) when $A_{V} \sim 0$, dropping rapidly to $B=0.3$ at $A_{V}=0.4$, in agreement with our results.

Furthermore, we find that neither the slope of the attenuation curve nor the UV bump depend on gas-phase metallicity (Figure 8, upper panels). For the slopes, this confirms, using a much larger sample, the results of Calzetti et al. (1994), where no systematic difference was found between the lower- and higher-metallicity galaxies in plots of UV power-law index versus the Balmer optical depth. This lack of dependence for both the slope and the bump places interesting constraints on the modeling of dust properties and for understanding the nature of the dust component responsible for the bump (e.g., Mathis 1994).

Overall, our results challenge the view, based on MW, LMC, and SMC extinction curves, that less massive galaxies have a weaker bump, supposedly due to a lower metallicity. The results (Figure 3) also prompt us to reconsider the idea that galaxies with high sSFRs have a UV radiation field that lead to the destruction of the carriers of the UV bump (Fischera \& Dopita 2011). According to this scenario, the UV bump may be prominent in normal SF galaxies but not in starbursts. The absence of the bump in starbursts is supported by a nondetection of a strong (MW- or LMC-like) bump in the Calzetti et al. (1994) analysis of IUE UV spectra. However, it is notable that the UV bump is apparently absent also in the IUE UV spectra of more normal SF galaxies from Kinney et al. (1993) parent sample. Out of the 140 galaxies in the Kinney atlas, only one shows a somewhat prominent UV bump feature (NGC 7714), a galaxy that happens to be a starburst. The IUE UV spectra tend to be noisy in the NUV, while the expected effect from the bumps we find is quite moderate (one-third of MW), which may explain the difficulty in detecting the bump in local galaxies. On the other hand, the stacking of high-S/N observed-frame optical spectra of Noll et al. (2007, 2009), for their sample of exquisitely bursty galaxies (log sSFR $\sim-8$ ), reveals strong evidence for a moderate UV bump in one-third of their $1<z<2.5$ sample, in agreement with our results.

While a growing number of studies show that average attenuation curves are steep and moderately bumpy at lower and higher redshifts, the question remains as to why we find that local starbursts (high-redshift analogs) have even steeper curves than "normal" star-forming galaxies, in clear contrast to the shallow Calzetti curve. We propose that there is a systematic difference in the slopes derived by "model-based" and by "comparison" methods, especially if the latter are based on the use of the Balmer decrement as a proxy for stellar attenuation.

First, in order to check whether the steep slope of our highredshift analogs is due to the differences in samples, we perform IR-luminosity-constrained SED fitting on Calzetti et al. (2000) galaxies that lie in the SDSS DR10 footprint and have requisite photometry from GALEX and WISE. We take the UV and optical photometry, as well as redshifts, from the NASA Sloan Atlas (v0_1_2; Blanton et al. 2011), which, by collecting redshifts from a variety of sources (including IPAC NED) has a more complete redshift coverage at $z<0.05$ than the SDSS spectroscopic catalog alone. Furthermore, the NASA Sloan Atlas photometry of SDSS images is optimized for extended objects. The resulting sample contains 14 out of the 33 galaxies used in Calzetti et al. (2000). On average, these galaxies lie 1 dex above the main sequence, similar to 
our high- $z$ analogs. The resulting curves of these 14 galaxies are steep $(\delta=-0.56 \pm 0.4$, or $n=1.3 \pm 0.4$ for the average and the $1 \sigma$ scatter), steeper on average than the SMC curve, and even slightly steeper than the curve of our high-redshift analogs shown in Figure 11. The average of the total to selective extinction in $V$ is $R_{V}=2.74$. The UV bump that we find for this sample is very modest (somewhat less pronounced than for our high- $z$ analogs), in qualitative agreement with Calzetti et al. (1994).

The reasons for the large discrepancy in slopes with respect to Calzetti et al. (2000) for the same sample are probably methodological rather than stemming from the data used (e.g., integrated photometry versus spectroscopy). As mentioned in Section 1, the method of Calzetti et al. (1994) consists of a comparison of UV/optical spectra of galaxies having different levels of attenuation, as determined from the nebular emission (the Balmer decrement). Once normalized to $E(B-V)=1$, this method produces the selective curve $\left(k_{\lambda}-R_{V}\right.$; Section 3.3). The total curve requires the determination of $R_{V}$ through the use of IR data (i.e., the energy balance) or by referring to nearly unattenuated near-IR photometry. The total curve for the Calzetti et al. (1994) sample has been established subsequently, in Calzetti et al. (2000), by keeping the selective curve the same as in the original work and solving for $R_{V}$.

We point out two issues relevant to the comparison method of Calzetti et al. (1994) that may be the source of this discrepancy; of these two we believe the second is more important. The comparison method looks at the SEDs of reddened galaxies with respect to the SEDs of galaxies that are considered to be dust free. Galaxies are selected as unreddened usually based on the nebular Balmer decrement, as a proxy for continuum attenuation. First, as pointed out by Wild et al. (2011) and Chevallard et al. (2013), the underlying assumption of the comparison method is that the attenuation curves do not vary with the dust content, as characterized, for example, by the optical opacity $\left(\tau_{V}\right.$ or $\left.A_{V}\right)$. The validity of this assumption has not been fully established in previous studies. Our results (Figure 5) and the theoretical analysis of Chevallard et al. (2013) suggest that this requirement is not fulfilled. Because in the comparison method the high-opacity (dusty) galaxies have greater leverage in the derivation of the curve (with the weight being proportional to $\Delta \tau_{V}$; Wild et al. 2011), the resulting attenuation curve will be biased toward the shallower slopes of high-opacity galaxies.

The second, and likely more significant, factor is that the comparison method relies on the use of nebular attenuation (the Balmer decrement) to place galaxies in different attenuation categories. However, the nebular extinction is a poor proxy for continuum attenuation, due to a high intrinsic dispersion between these quantities, as demonstrated in Figure 12. This potential issue with the comparison method was pointed out by Charlot \& Fall (2000). Based on SDSS emission-line data, we find that the galaxies with $\tau_{\text {Balmer }}<0.1$ (which would be considered unattenuated) actually have a significant average continuum attenuation of $A_{\mathrm{FUV}}=1.3$ (Figure 12). Likewise, the galaxies considered to be highly attenuated according to the Balmer decrement $\left(\tau_{\text {Balmer }} \sim 0.7\right)$ have an average $A_{\mathrm{FUV}}=$ 3.0 , well below that of actual most opaque galaxies $\left(A_{\mathrm{FUV}} \sim 4.5\right)$. Thus ,the difference in attenuations between galaxies selected as dusty and the ones assumed to be dust free is smaller by more than a factor of two than what is assumed based on the Balmer

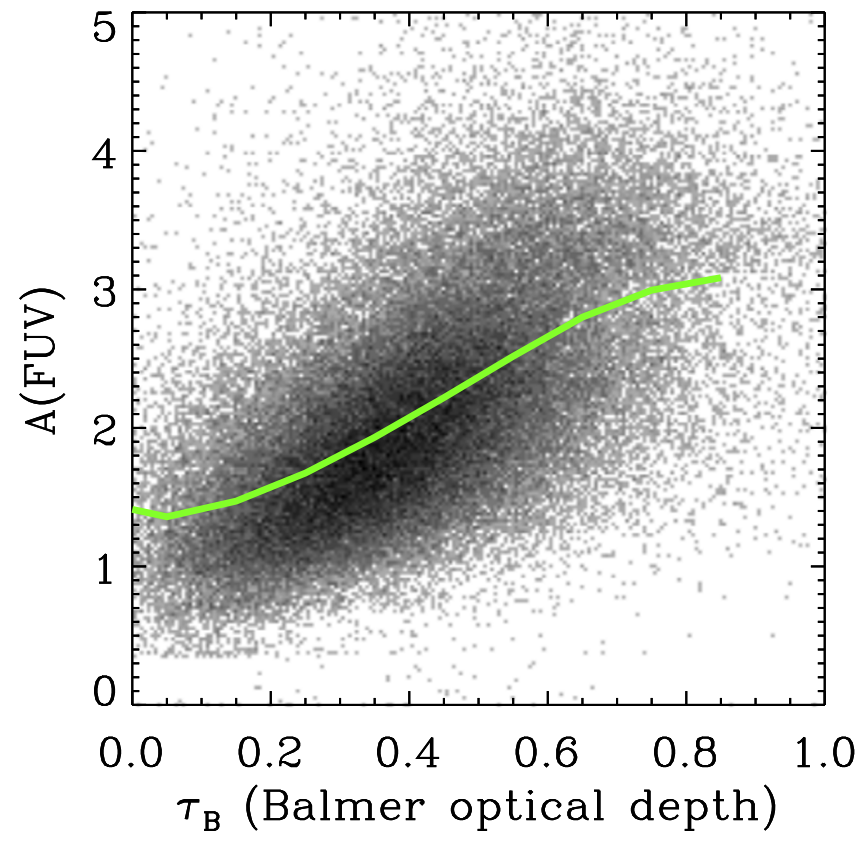

Figure 12. Comparison of attenuation affecting the FUV continuum light and the Balmer optical depth affecting the $\mathrm{H}$ II regions. While the two attenuations are correlated, there is a large degree of intrinsic scatter between the two, such that the identification of unreddened galaxies using the Balmer decrement results in galaxies that on average have a substantial dust attenuation (1.3 mag in FUV). FUV attenuation is derived from our SED fitting and has a typical error of $0.3 \mathrm{mag}$ (i.e., it does not dominate the scatter), whereas the Balmer decrement optical depths are based on SDSS spectra. The green curve represents the trend of binned averages.

decrement. Deriving the attenuation curve by dividing the SED of a reddened galaxy by an SED of a galaxy that is not truly unreddened will result in a shallower slope.

More detailed investigation of the systematics resulting from the methodology will be important for future work. Interestingly, other studies that use the comparison method based on Balmer decrements also obtain shallow slopes of dust curves (either selective or absolute), regardless of whether the population consists of starbursting or more normal galaxies. Our results do agree on the point that there is no large difference in the average curves of normal and starbursting (and likely high-redshift) galaxies, but here we find that those curves are, on average, systematically steeper, consistent with other studies that are based on model-based approaches.

\section{Summary}

This paper presents constraints on dust attenuation curves for a very large sample of galaxies in the local universe, allowing for detailed statistical characterization. Our main findings are as follows:

1. Galaxies in the local universe exhibit a wide range of dust attenuation curve slopes (power-law exponent range $\sim 1$ ), from shallow slopes similar to the slope of the Calzetti curve to slopes significantly steeper than the SMC extinction curve.

2. On average, local star-forming galaxies have steep curves, almost as steep as the SMC extinction curve.

3 . The steepness of the curves is most strongly correlated with the optical opacity $\left(A_{V}\right)$, with higher opacity 
implying a shallower curve, confirming the Leja et al. (2017) results and in agreement with the predictions of radiation transfer models with realistic geometry (Chevallard et al. 2013).

4. Slopes tend to be shallower in more massive galaxies, but this trend is almost entirely the consequence of the fact that more massive galaxies have higher $A_{V}$.

5. Slopes have a secondary dependence on SFR, such that the galaxies away from the star-forming sequence in either direction (toward starbursts and toward quiescent galaxies) have somewhat steeper slopes than galaxies of the same mass closer to the main sequence.

6. Consequently, the analogs of high-redshift galaxies have, on average, somewhat steeper curves than the normal star-forming galaxies of the same mass, i.e., similar to or steeper than the SMC curve.

7. Galaxies exhibit a range of bump strengths, but rarely exceeding the MW value. Stronger bumps tend to be found in galaxies with steeper curves, as previously found at higher redshifts (Kriek \& Conroy 2013), but the correlation is not tight. On average, the contribution of the bump to the NUV attenuation is relatively small ( $\sim 1 / 3$ of that of the MW bump), and can be, to first order, ignored, as it affects the NUV magnitude by only $0.1 \mathrm{mag}$.

8. Neither the slopes nor the bump strengths have a strong dependence on gas metallicity.

9. Galaxies above $\log M_{*}=10$ have attenuation curve slopes that exhibit a moderate dependence on galaxy inclination for $b / a>0.6$, which, as in the case of mass dependence, is fundamentally due to the dependence of the attenuation curve slope on optical opacity.

We also present functional fits for dust attenuation curves suitable for use in low- and high-redshift studies. The current work has significant implications for the study of IRX- $\beta$ and $A_{\mathrm{FUV}}-\beta$ relations (e.g., Boquien et al. 2009, 2012) and will be the subject of a forthcoming publication.
The catalog of IR luminosity-constrained SED-fitting parameters used in this work, such as the stellar mass, dust attenuation, and the SFR, are publicly released as GSWLC- 2 .

The construction of GSWLC was funded through NASA ADAP award NNX12AE06G. We thank Daniella Calzetti and Veronique Buat for valuable discussions. Funding for SDSS-III has been provided by the Alfred P. Sloan Foundation, the Participating Institutions, the National Science Foundation, and the U.S. Department of Energy Office of Science. The SDSS-III Web site is http://www.sdss3.org/. Based on observations made with the NASA Galaxy Evolution Explorer (GALEX). GALEX is operated for NASA by the California Institute of Technology under NASA contract NAS5-98034. This publication makes use of data products from the Widefield Infrared Survey Explorer, which is a joint project of the University of California, Los Angeles, and the Jet Propulsion Laboratory/California Institute of Technology, funded by the National Aeronautics and Space Administration.

\section{Appendix}

\section{A.1. Mock SED Fitting}

In order to assess whether the choice of priors and the potentially limited constraining power of the data lead to significant systematics in the derived values of the attenuation curve slope and UV bump amplitude, we perform, using CIGALE, mock SED fitting in which we take the photometry of the best-fitting model from the nominal run and repeat the SED fitting with this photometry instead of the actual one, while using the actual photometry errors (Salim et al. 2009). The model photometry, which is treated as the input photometry in the mock fitting, has associated "true" physical parameters that are known. Mock fitting allows us to see how well these parameters are recovered. The results are shown in Figure 13 for the effective FUV attenuation $\left(A_{\mathrm{FUV}}\right)$, slope deviation $(\delta)$, and UV bump amplitude $(B)$. True values of the slope and the bump are highly discrete $(0.2$ and 2$)$, so we
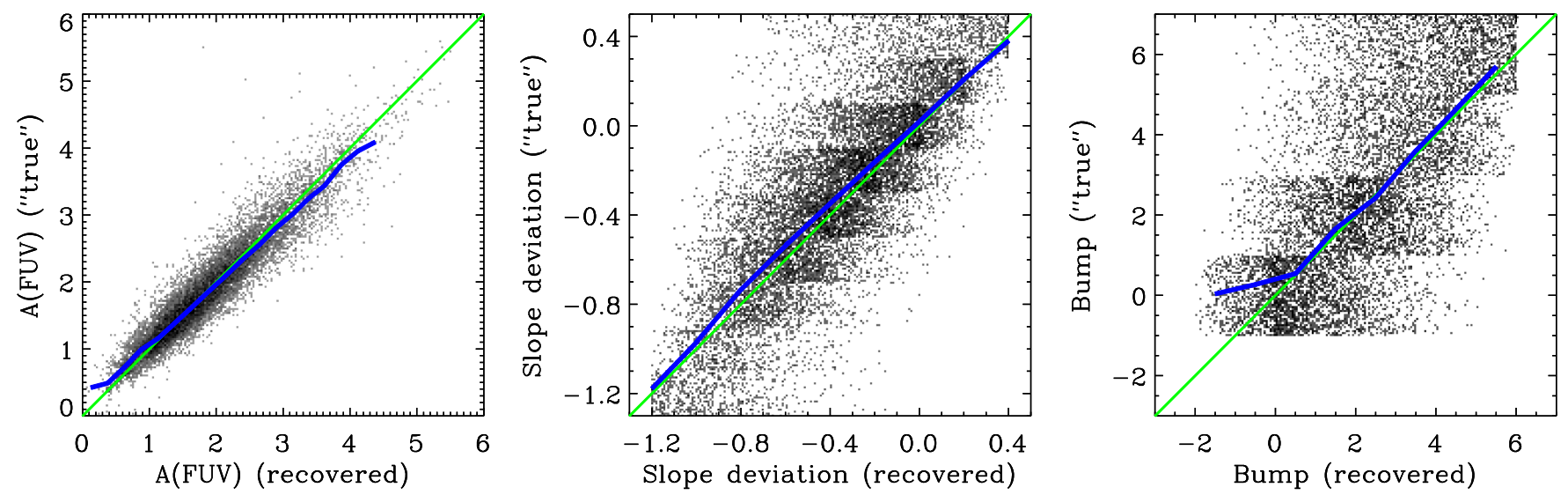

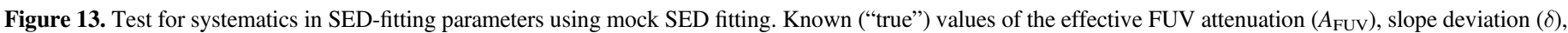

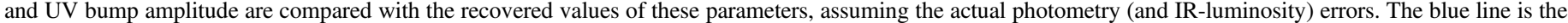
running median, while the green line is a 1:1 relation. The dispersion is indicative of the parameter errors. $(B)$ 
perturb them by a random value to show the spread. For the bump amplitude, we show only the points with a physical true bump amplitude $(B>0)$. Notably, there are no significant systematics in the recovered values.

\section{ORCID iDs}

Samir Salim (i) https://orcid.org/0000-0003-2342-7501 Médéric Boquien (i) https://orcid.org/0000-0003-0946-6176 Janice C. Lee (10) https://orcid.org/0000-0002-2278-9407

\section{References}

Arnouts, S., Le Floc'h, E., Chevallard, J., et al. 2013, A\&A, 558, A67 Baldwin, J. A., Phillips, M. M., \& Terlevich, R. 1981, PASP, 93, 5 Battisti, A. J., Calzetti, D., \& Chary, R.-R. 2016, ApJ, 818, 13 Battisti, A. J., Calzetti, D., \& Chary, R.-R. 2017, ApJ, 840, 109 Bauer, A. E., Drory, N., Hill, G. J., \& Feulner, G. 2005, ApJL, 621, L89 Blanton, M. R., Kazin, E., Muna, D., Weaver, B. A., \& Price-Whelan, A. 2011, AJ, 142, 31

Boquien, M., Buat, V., Boselli, A., et al. 2012, A\&A, 539, A145

Boquien, M., Calzetti, D., Kennicutt, R., et al. 2009, ApJ, 706, 553

Bourne, N., Dunne, L., Maddox, S. J., et al. 2016, MNRAS, 462, 1714

Bouwens, R. J., Illingworth, G. D., Oesch, P. A., et al. 2014, ApJ, 793, 115

Bowler, R. A. A., Dunlop, J. S., McLure, R. J., et al. 2015, MNRAS, 452, 1817

Bromage, G. E., \& Nandy, K. 1983, MNRAS, 204, 29P

Bruzual, G., \& Charlot, S. 2003, MNRAS, 344, 1000

Buat, V., Noll, S., Burgarella, D., et al. 2012, A\&A, 545, A141

Burgarella, D., Buat, V., \& Iglesias-Páramo, J. 2005, MNRAS, 360, 1413

Calzetti, D. 2001, PASP, 113, 1449

Calzetti, D., Armus, L., Bohlin, R. C., et al. 2000, ApJ, 533, 682

Calzetti, D., Kinney, A. L., \& Storchi-Bergmann, T. 1994, ApJ, 429, 582

Cardelli, J. A., Clayton, G. C., \& Mathis, J. S. 1988, ApJL, 329, L33

Cardelli, J. A., Clayton, G. C., \& Mathis, J. S. 1989, ApJ, 345, 245

Chabrier, G. 2003, PASP, 115, 763

Charlot, S., \& Fall, S. M. 2000, ApJ, 539, 718

Chary, R., \& Elbaz, D. 2001, ApJ, 556, 562

Chevallard, J., Charlot, S., Wandelt, B., \& Wild, V. 2013, MNRAS, 432, 2061

Conroy, C. 2013, ARA\&A, 51, 393

Conroy, C., Schiminovich, D., \& Blanton, M. R. 2010, ApJ, 718, 184

Conroy, C., White, M., \& Gunn, J. E. 2010, ApJ, 708, 58

da Cunha, E., Charlot, S., \& Elbaz, D. 2008, MNRAS, 388, 1595

Davé, R., Rafieferantsoa, M. H., Thompson, R. J., \& Hopkins, P. F. 2017, MNRAS, 467, 115

Fanelli, M. N., O'Connell, R. W., \& Thuan, T. X. 1988, ApJ, 334, 665

Ferland, G. J., Korista, K. T., Verner, D. A., et al. 1998, PASP, 110, 761

Finkelstein, S. L., Ryan, R. E., Jr., Papovich, C., et al. 2015, ApJ, 810, 71

Fischera, J., \& Dopita, M. 2011, A\&A, 533, A117

Fitzpatrick, E. L. 1986, AJ, 92, 1068

Fitzpatrick, E. L., \& Massa, D. 1986, ApJ, 307, 286

Fitzpatrick, E. L., \& Massa, D. 1988, ApJ, 328, 734

Gordon, K. D., Clayton, G. C., Misselt, K. A., Landolt, A. U., \& Wolff, M. J. 2003, ApJ, 594, 279

Guzmán, R., Gallego, J., Koo, D. C., et al. 1997, ApJ, 489, 559

Hagen, L. M. Z., Siegel, M. H., Hoversten, E. A., et al. 2017, MNRAS, 466, 4540
Hayward, C. C., \& Smith, D. J. B. 2015, MNRAS, 446, 1512

Holwerda, B. W., Böker, T., Dalcanton, J. J., Keel, W. C., \& de Jong, R. S. 2013, MNRAS, 433, 47

Iglesias-Páramo, J., Boselli, A., Gavazzi, G., \& Zaccardo, A. 2004, A\&A, 421,887

Inoue, A. K. 2001, AJ, 122, 1788

Inoue, A. K. 2011, MNRAS, 415, 2920

Juneau, S., Bournaud, F., Charlot, S., et al. 2014, ApJ, 788, 88

Kauffmann, G., Heckman, T. M., Tremonti, C., et al. 2003, MNRAS, 346, 1055

Keel, W. C. 1993, in ASP Conf. Ser., Vol. 35, Massive Stars: Their Lives in the Interstellar Medium 35, ed. J. P. Cassinelli \& E. B. Churchwell (San Francisco, CA: ASP), 498

Keel, W. C., Manning, A. M., Holwerda, B. W., Lintott, C. J., \& Schawinski, K. 2014, AJ, 147, 44

Kewley, L. J., \& Dopita, M. A. 2002, ApJS, 142, 35

Kinney, A. L., Bohlin, R. C., Calzetti, D., Panagia, N., \& Wyse, R. F. G. 1993 , ApJS, 86, 5

Kriek, M., \& Conroy, C. 2013, ApJL, 775, L16

Lang, D., Hogg, D. W., \& Schlegel, D. J. 2016, AJ, 151, 36

Leitherer, C., Li, I.-H., Calzetti, D., \& Heckman, T. M. 2002, ApJS, 140, 303

Leja, J., Johnson, B. D., Conroy, C., van Dokkum, P. G., \& Byler, N. 2017, ApJ, 837, 170

Maraston, C. 2005, MNRAS, 362, 799

Mathis, J. S. 1994, ApJ, 422, 176

Nandy, K., Morgan, D. H., Willis, A. J., et al. 1980, Natur, 283, 725

Nandy, K., Thompson, G. I., Jamar, C., Monfils, A., \& Wilson, R. 1975, A\&A, 44, 195

Noll, S., Burgarella, D., Giovannoli, E., et al. 2009, A\&A, 507, 1793

Noll, S., Pierini, D., Pannella, M., \& Savaglio, S. 2007, A\&A, 472, 455

O’Donnell, J. E. 1994, ApJ, 422, 158

Oesch, P. A., Bouwens, R. J., Illingworth, G. D., et al. 2014, ApJ, 786, 108

Peek, J. E. G., \& Schiminovich, D. 2013, ApJ, 771, 68

Pérez-González, P. G., Gil de Paz, A., Zamorano, J., et al. 2003, MNRAS, 338,525

Prevot, M. L., Lequeux, J., Prevot, L., Maurice, E., \& Rocca-Volmerange, B. 1984, A\&A, 132, 389

Reddy, N. A., Kriek, M., Shapley, A. E., et al. 2015, ApJ, 806, 259

Reddy, N. A., Oesch, P. A., Bouwens, R. J., et al. 2018, ApJ, 853, 56

Rocca-Volmerange, B., Prevot, L., Prevot-Burnichon, M. L., Ferlet, R., \& Lequeux, J. 1981, A\&A, 99, L5

Salim, S., Dickinson, M., Rich, R. M., et al. 2009, ApJ, 700, 161

Salim, S., Lee, J. C., Davé, R., \& Dickinson, M. 2015, ApJ, 808, 25

Salim, S., Lee, J. C., Janowiecki, S., et al. 2016, ApJS, 227, 2

Salim, S., Rich, R. M., Charlot, S., et al. 2007, ApJS, 173, 267

Salmon, B., Papovich, C., Long, J., et al. 2016, ApJ, 827, 20

Seaton, M. J. 1979, MNRAS, 187, 73P

Smit, R., Bouwens, R. J., Labbé, I., et al. 2014, ApJ, 784, 58

Stebbins, J., \& Whitford, A. E. 1943, ApJ, 98, 20

Stecher, T. P. 1965, ApJ, 142, 1683

Tremonti, C. A., Heckman, T. M., Kauffmann, G., et al. 2004, ApJ, 613, 898

Tress, M., Mármol-Queraltó, E., Ferreras, I., et al. 2018, MNRAS, 475, 2363

Valiante, E., Smith, M. W. L., Eales, S., et al. 2016, MNRAS, 462, 3146

Viaene, S., Sarzi, M., Baes, M., Fritz, J., \& Puerari, I. 2017, MNRAS, 472, 1286

White, R. E., III, \& Keel, W. C. 1992, Natur, 359, 129

Wild, V., Charlot, S., Brinchmann, J., et al. 2011, MNRAS, 417, 1760

Yuan, H. B., Liu, X. W., \& Xiang, M. S. 2013, MNRAS, 430, 2188 